\title{
A Physics-guided Coordinated Distributed MPC Method for Shape Control of an Antenna Reflector
}

\author{
Fei Li, Student Member, IEEE, Haijun Peng, Member, IEEE, Xiangshuai Song, Jinguo Liu, Senior \\ Member, IEEE, Shujun Tan, and Zhaojie Ju, Senior Member, IEEE
}

\begin{abstract}
Active shape control for an antenna reflector is a significant procedure used to compensate the impacts for a complicated space environment. In this paper, a physics-guided distributed model predictive control (DMPC) framework for reflector shape control with input saturation is proposed. First, guided by the actual physical characteristics, an overall structural system is decomposed into multilevel subsystems with the help of a so-called substructuring technique. For each subsystem, a prediction model with information interaction is discretized by an explicit Newmark- $\beta$ method. Then, to improve the systemwide control performance, a coordinator among all the subsystems is designed in an iterative fashion. The input saturation constraints are addressed by transforming the original problem into a linear complementarity problem (LCP). Finally, by solving the LCP, the input trajectory can be obtained. The performance of the proposed DMPC algorithm is validated through an experiment on the shape control of an antenna reflector structure.
\end{abstract}

Index Terms - Shape control, distributed model predictive control (DMPC), substructuring technique, input saturation, linear complementarity problem (LCP).

\section{INTRODUCTION}

I $\mathrm{N}$ structural shape control, one intends to seek a suitable control law so that the structural displacement field distorted from its original shape ultimately vanishes, or so that the structure follows certain expected field of trajectories [1]. The surface shape control of an antenna reflector, which has extensive applications such as satellite communications and deep space exploration, is a typical topic in the structural shape control field. To achieve the needed surface accuracy of an antenna reflector, certain high-precision active shape control strategies have been proposed.

As early as the 1980s, using temperature gradients, Haftka and Adelman proposed an analytical procedure for the static shape control of flexible structures with applications to space

Manuscript received xxx, $\mathrm{xxx}$.; revised $\mathrm{xxxx}$, xxx; accepted $\mathrm{xxx}, \mathrm{xxxx}$. This work was supported in part by the National Science Foundation of China under Grant 11922203, Grant 11772074, Grant 11572069 and Grant 51775541, in part by the National Key R\&D Program of China under Grant 2018YFB1304600, and in part by the CAS Interdisciplinary Innovation Team under Grant JCTD-2018-11. (Corresponding author: Haijun Peng)

F. Li and H. Peng are with the Department of Engineering Mechanics, State Key Laboratory of Structural Analysis of Industrial Equipment, Dalian University of Technology, Dalian 116024, China (e-mail: lif2014@mail.dlut. edu.cn; hjpeng(d)dut.edu.cn ). antennas [2]. Thereafter, based on the experimental influence coefficient matrix (ICM) model, Song et al. presented an error least-squares method for shape control of an antenna reflector equipped with piezoelectric (PZT) actuators [3]. Xie et al. developed a gain scheduling method coupled with the $\mathrm{H}_{\infty}$ robust control to maintain the shape accuracy of a deployable mesh reflector [4]. All the above controllers were designed as a single centralized controller, which is usually applicable for smallscale structures with few actuators. However, in practical applications, controlled structural systems may naturally be so large and have so many inputs and outputs that conventional centralized control strategies would fail to give credible solutions due to either a lack of either centralized information collection ability or computing ability. Moreover, a centralized controller lacks fault tolerance; once the single centralized controller fails, the whole system will lose control. Therefore, drawing on the concept of "divide and conquer", the distributed control framework [5]-[7], where the overall system is divided and controlled by a series of local regulators independently, has been widely used for addressing the aforementioned difficulties.

The notion of distributed control was proposed in the $1970 \mathrm{~s}$ [6]. This control strategy has the common characteristics of less computation burden, high fault tolerance, and flexibility in the system structure [8]. The important and pioneering work on distributed control dates back to the early contributions in the reference [9], which studied the problem of stabilizing a decentralized linear time-invariant control system via local feedback with dynamic compensation. Thereafter, a variety of control approaches combined with the distributed concept have been reported [10]-[15], but there are few studies on the shape control of smart structural systems. In addition, because the physical inputs of actuators in practical applications are usually limited, so-called input saturation must be considered in controller design. In this paper, model predictive control (MPC)

X. Song and S. Tan are with the school of Aeronautics and Astronautics, Dalian University of Technology, Dalian 116024, China (e-mail: songxiangshuai@mail.dlut.edu.cn; tansj@dlut.edu.cn).

J. Liu is with the State Key Laboratory of Robotics, Shenyang Institute of Automation, Institutes for Robotics and Intelligent Manufacturing, Chinese Academy of Sciences, Shenyang 110016, China (e-mail: liujinguo@sia.cn).

Z. Ju is with the School of Computing, University of Portsmouth, PO1 3HE Portsmouth, U.K. (e-mail: Zhaojie.Ju@port.ac.uk ). 
[16]-[18], a well-established method for large multivariable constrained control problems, will be introduced as local regulators. Then, inspired by the main concept of distributed control, a novel DMPC framework will be proposed and applied to the smart structural shape control.

Previous work has been done to improve the control performance of DMPC. Scattolini [19] classified the DMPC methods from different perspectives, involving the topology of communication network, the protocol of information exchange among regulators, and the type of cost function. Camponogara et al. [20] proposed a DMPC method with a certain degree of coordination between each MPC controller that was designed by locally relevant variables, cost and constraints. Conte et al. [21] presented a new distributed synthesis method for stabilizing the cooperative DMPC networks of linear systems. In all of the above literature, research has made significant contributions on controller design, closed-loop stability, and information interactions for the DMPC method. However, all these contributions were based on a premise, i.e., the system decomposition mode, which is an important foundation in the distributed control method. Some distributed control algorithms for a system that can be divided at different time scales have been proposed [22], [23]. However, the time-scale-based decomposition modes in these references are inappropriate for flexible structural systems because structural systems are usually analyzed on the same discrete time scale. This work will break through the limitations of all the existing decomposition modes for distributed systems in theory.

From a physics-guided point of view, i.e., based on the physical characteristics of a structure, substructuring technology [24], [25], which has been extensively used for solving large-scale systems in structural mechanics, can be adopted to decompose an entire structural system into a series of subsystems. First, the entire system is divided into several independent subsystems by finite element mesh generation, and the element mesh grid of this partitioned system is considered the first-level grid. Then, for each divided subsystem, two types of nodes are defined, i.e., the internal and boundary nodes, which are independent of and connected with the nodes of other adjacent subsystems. Finally, the Schur complement method [26] is applied to eliminate the coordinates of internal nodes, and the remaining boundary nodes are treated as a second-level grid, which can be further decomposed into subsystems. In a similar fashion, other multilevel grids can be established.

Inspired by the main concept of the aforementioned physicsguided concept and distributed control strategy, this paper proposes a physics-guided DMPC framework for the shape control of linear structural systems. In the proposed DMPC framework, each local MPC controller can be designed flexibly at different levels according to the topological shape, actuator arrangement, and calculation scale of a structural system; the state evolution is assumed to be affected by both the states and inputs of other interacting subsystems. Furthermore, to improve the global performance of the DMPC, a coordinator is designed among all the distributed controllers in an iterative fashion. At convergence, the proposed DMPC algorithm can obtain optimal (centralized) control performance. In addition, the aforementioned input saturation constraints will be considered by introducing the Lagrange multipliers and extending the local cost function of each MPC controller; then, by transforming the original problem into a series of LCPs, the constraints can be directly satisfied by solving these LCPs. The performance of the proposed DMPC method is validated by an experiment on the shape control of an antenna reflector. The contributions of this work are summarized as follows:

1) (system decomposition mode) Based on the substructuring technique, a physics-guided DMPC method with a novel multilevel system decomposition mode is proposed. The local MPC controllers for each subsystem can be designed flexibly at different levels.

2) (online optimization strategy) The original online constrained optimization problem of DMPC is transformed into an LCP so that the input saturation constraints can be satisfied directly. In addition, a coordinator is designed in an iterative fashion, so the control performance of the distributed controllers can be close to that of the centralized controller.

3) (experimental evaluation) The feasibility, effectiveness, flexibility, and fault tolerance of the proposed method are verified by an experiment on the shape control of an antenna reflector structure. The proposed DMPC algorithm is also applicable to the control problems of other linear time-invariant structural systems.

The rest of this paper is structured as follows. In section II, the primary formulation of shape control problem for structural systems is introduced. Based on the substructuring technique, the overall system is decomposed into multilevel subsystems in section III. Then, in section IV, a physics-guided coordinated DMPC algorithm framework for structural shape control is proposed in detail. The control performance of the proposed DMPC controller is illustrated by an experiment on the shape control of an antenna reflector structure in section V. Finally, some concluding remarks are given in section VI.

\section{PROBlem Formulation}

Considering a linear smart antenna reflector, a multilevel DMPC controller is designed with the help of PZT actuators to minimize the deformation of the structure from its ideal shape.

A dynamic model of the reflector structure, including the active PZT actuator elements [27] and other structural elements such as the composite shell and thin plate, can be constructed by the finite element method (FEM). The governing equation of the antenna reflector structure is given as

$$
\boldsymbol{M} \ddot{\boldsymbol{x}}+\boldsymbol{C} \dot{\boldsymbol{x}}+\boldsymbol{K} \boldsymbol{x}=\boldsymbol{D u}
$$

where $\boldsymbol{M} \in \mathbb{R}^{n x n}$ and $\boldsymbol{K} \in \mathbb{R}^{n x n}$ are the mass matrix and the stiffness matrix of the system, respectively; $\boldsymbol{C} \in \mathbb{R}^{n \times n}$ is the Rayleigh damping matrix, that is, $\boldsymbol{C}=\gamma \boldsymbol{M}+\beta \boldsymbol{K}$, where $\gamma$ and $\beta$ are constant coefficients; $\boldsymbol{D} \in \mathbb{R}^{n \times m}$ is the location matrix of all the control input vector $\boldsymbol{u} \in \mathbb{R}^{m \times 1}$ (i.e., the control voltages of PZT actuators); $\boldsymbol{x} \in \mathbb{R}^{n \times 1}, \dot{\boldsymbol{x}} \in \mathbb{R}^{n \times 1}$ and $\ddot{\boldsymbol{x}} \in \mathbb{R}^{n \times 1}$ are the nodal displacement, velocity, and acceleration vectors, respectively, of the antenna reflector in global coordinate.

Then, decomposing the overall system into $S$ interconnected subsystems, the decentralized model for any subsystem $i$ with 
$n_{i}$ degrees of freedom and $m_{i}$ actuators can be given as

$$
\tilde{\boldsymbol{M}}_{i i} \ddot{\boldsymbol{x}}_{i i}+\tilde{\boldsymbol{C}}_{i i} \dot{\boldsymbol{x}}_{i i}+\tilde{\boldsymbol{K}}_{i i} \boldsymbol{x}_{i i}=\tilde{\boldsymbol{D}}_{i i} \boldsymbol{u}_{i}
$$

To introduce the impact of other interacting subsystem $j \neq i$ on subsystem $i$ and retain the most advantages of the decentralized modeling framework, the distributed dynamic model of the $i^{\text {th }}$ subsystem, in this work, is defined as

$$
\hat{\boldsymbol{M}}_{i} \ddot{\boldsymbol{x}}_{i}+\hat{\boldsymbol{C}}_{i} \dot{\boldsymbol{x}}_{i}+\hat{\boldsymbol{K}}_{i} \boldsymbol{x}_{i}=\boldsymbol{D}_{i i} \boldsymbol{u}_{i}+\sum_{j \neq i}^{S} \boldsymbol{D}_{i j} \boldsymbol{u}_{j}
$$

where $i, j \in\{1, S\}, S$ denotes the number of subsystems, the notation $\{1, S\}$ represents the sequence of integers $1,2, \ldots, S$, and

$$
\begin{array}{ll}
\hat{\boldsymbol{M}}_{i}=\operatorname{diag}\left(\tilde{\boldsymbol{M}}_{i 1}, \tilde{\boldsymbol{M}}_{i 2}, \cdots, \tilde{\boldsymbol{M}}_{i i}, \cdots, \tilde{\boldsymbol{M}}_{i S}\right) & \ddot{\boldsymbol{x}}_{i}=\left\{\ddot{\boldsymbol{x}}_{i 1}^{\mathrm{T}}, \cdots, \ddot{\boldsymbol{x}}_{i i}^{\mathrm{T}}, \cdots, \ddot{\boldsymbol{x}}_{i s}^{\mathrm{T}}\right\}^{\mathrm{T}} \\
\hat{\boldsymbol{C}}_{i}=\operatorname{diag}\left(\tilde{\boldsymbol{C}}_{i 1}, \tilde{\boldsymbol{C}}_{i 2}, \cdots, \tilde{\boldsymbol{C}}_{i i}, \cdots, \tilde{\boldsymbol{C}}_{i S}\right) & \dot{\boldsymbol{x}}_{i}=\left\{\dot{\boldsymbol{x}}_{i 1}^{\mathrm{T}}, \cdots, \dot{\boldsymbol{x}}_{i i}^{\mathrm{T}}, \cdots, \dot{\boldsymbol{x}}_{i s}^{\mathrm{T}}\right\}^{\mathrm{T}} \\
\hat{\boldsymbol{K}}_{i}=\operatorname{diag}\left(\tilde{\boldsymbol{K}}_{i 1}, \tilde{\boldsymbol{K}}_{i 2}, \cdots, \tilde{\boldsymbol{K}}_{i i}, \cdots, \tilde{\boldsymbol{K}}_{i S}\right) & \boldsymbol{x}_{i}=\left\{\boldsymbol{x}_{i 1}^{\mathrm{T}}, \cdots, \boldsymbol{x}_{i i}^{\mathrm{T}}, \cdots, \boldsymbol{x}_{i s}^{\mathrm{T}}\right\}^{\mathrm{T}} \\
\boldsymbol{D}_{i j}=\left\{\boldsymbol{0}_{m_{j} \times n_{i 1}}, \cdots, \mathbf{0}_{m_{j} \times n_{i j(j-1)}}, \tilde{\boldsymbol{D}}_{i j}^{\mathrm{T}}, \mathbf{0}_{m_{j} \times n_{i(j+1)}}, \cdots, \mathbf{0}_{m_{j} \times n_{i i}}\right\}^{\mathrm{T}}
\end{array}
$$

Let state vector $\boldsymbol{z}_{i}^{\mathrm{T}}=\left\{\boldsymbol{x}_{i}^{\mathrm{T}}, \dot{\boldsymbol{x}}_{i}^{\mathrm{T}}\right\}$; then, the above equation (3) can be transformed into the state-space formulation, given as

$$
\begin{gathered}
\dot{\boldsymbol{z}}_{i}=\boldsymbol{A}_{i i} \boldsymbol{z}_{i}+\boldsymbol{B}_{i i} \boldsymbol{u}_{i}+\sum_{\mathrm{j} \neq 1}^{S} \boldsymbol{B}_{i j} \boldsymbol{u}_{j} \\
\boldsymbol{A}_{i i}=\left[\begin{array}{cc}
\mathbf{0} & \boldsymbol{I} \\
-\hat{\boldsymbol{M}}_{i}^{-1} \hat{\boldsymbol{K}}_{i} & -\hat{\boldsymbol{M}}_{i}^{-1} \hat{\boldsymbol{C}}_{i}
\end{array}\right], \boldsymbol{B}_{i i}=\left[\begin{array}{c}
\mathbf{0} \\
\hat{\boldsymbol{M}}_{i}^{-1} \boldsymbol{D}_{i i}
\end{array}\right], \boldsymbol{B}_{i j}=\left[\begin{array}{c}
\mathbf{0} \\
\hat{\boldsymbol{M}}_{i}^{-1} \boldsymbol{D}_{i j}
\end{array}\right]
\end{gathered}
$$

A DMPC problem essentially solves a series of optimization problems in which the suboptimal inputs $\boldsymbol{u}_{i}(t)$ of each subsystem are optimized with respect to a local cost function $\varphi_{i}$. Consider the continuous-time control system (5) and assume that the prediction horizon time is $T$; then, a standard DMPC at sampling time $t_{k}$ can be formulated as follows:

Find:

$$
\boldsymbol{u}_{i}(t) \in \underset{\boldsymbol{u}_{i}(t)}{\arg \min } \varphi_{i}\left(\boldsymbol{y}_{i}(t), \boldsymbol{u}_{i}(t)\right), t \in\left[t_{k}, t_{k}+T\right]
$$

Subject to: $\quad \dot{\boldsymbol{z}}_{i}(t)=\boldsymbol{A}_{i i} \boldsymbol{z}_{i}(t)+\boldsymbol{B}_{i i} \boldsymbol{u}_{i}(t)+\sum_{j \neq i}^{S} \boldsymbol{B}_{i j} \boldsymbol{u}_{j}(t)$

$$
\begin{gathered}
\boldsymbol{y}_{i}(t)=\overline{\boldsymbol{C}}_{i} \boldsymbol{z}_{i}(t) \\
\boldsymbol{\mu}_{\min } \leq \boldsymbol{u}_{i}(t) \leq \boldsymbol{\mu}_{\max }
\end{gathered}
$$

With:

$$
\varphi_{i}=\frac{1}{2} \int_{t_{k}}^{t_{k}+T}\left(\left\|\boldsymbol{y}_{i}(\tau)-\boldsymbol{y}_{i d}\right\|_{\boldsymbol{Q}_{i}}^{2}+\left\|\boldsymbol{u}_{i}(\tau)\right\|_{\boldsymbol{R}_{i}}^{2}\right) \mathrm{d} \tau
$$

where in (11), the operator $\|*\|_{\Omega}^{2}$ denotes the square of a weighted Euclidean norm, i.e., $\|\boldsymbol{r}\|_{\boldsymbol{\Omega}}^{2}=\boldsymbol{r}^{T} \boldsymbol{\Omega} \boldsymbol{r} ; \boldsymbol{y}_{i d}$ (a constant vector in this paper) is the known target state; $\boldsymbol{y}_{i}$ is the output variable; and $\boldsymbol{Q}_{i} \geqslant 0$ and $\boldsymbol{R}_{i}>0$ are the nonnegative and positive definite symmetric weighting matrices, respectively. Equation (8) is the $i^{\text {th }}$ subsystem model, which is used for predicting the future state evolution of the subsystem with interactions of states and inputs. $\overline{\boldsymbol{C}}_{i}$ is the observation output coefficient matrix, which can be created based on the degrees of freedom of all the observation nodes in the $i^{\text {th }}$ subsystem. In addition, the input saturation constraint is described by inequality (10).

\section{MultileVel System Decomposition by A PHYSICS-GUIDED CONCEPT}

Before constructing a DMPC controller of a large-scale smart structural system, an important and unresolved issue is how to decompose the overall system model into a series of small subsystem models where each subsystem being controlled by an independent MPC controller [28]. In this section, inspired by a physics-guided concept, a multilevel decomposition mode based on the substructuring technique is introduced to divide the entire large-scale structure into several substructures. Subsequently, the prediction models of each subsystem can be established at different levels.

\section{A. Physics-guided Multilevel Decomposition Mode}

The overall dynamic model (1) can be solved by many mature numerical algorithms; herein, the classic Newmark- $\beta$ algorithm [29] is adopted. Thereafter, the novel multilevel decomposition strategy is presented as follows.

First, the overall structural system with implanted actuators is discretized by the finite element mesh generation technique. It is divided into $S$ decoupled subsystems by introducing the interior boundaries. Based on (1), the dynamic model of the $i^{\text {th }}$ decentralized subsystem can be given as

$$
\boldsymbol{M}_{i} \ddot{\boldsymbol{x}}_{i}+\boldsymbol{C}_{i} \dot{\boldsymbol{x}}_{i}+\boldsymbol{K}_{i} \boldsymbol{x}_{i}=\boldsymbol{D}_{i} \boldsymbol{u}_{i}
$$

In the Newmark- $\beta$ algorithm, it is assumed that the velocity and displacement of the system satisfy the following relationships

$$
\begin{gathered}
\dot{\boldsymbol{x}}_{i}\left(t_{k}\right)=\dot{\boldsymbol{x}}_{i}\left(t_{k-1}\right)+\left[(1-\delta) \ddot{\boldsymbol{x}}_{i}\left(t_{k-1}\right)+\delta \ddot{\boldsymbol{x}}_{i}\left(t_{k}\right)\right] \Delta t \\
\boldsymbol{x}_{i}\left(t_{k}\right)=\boldsymbol{x}_{i}\left(t_{k-1}\right)+\dot{\boldsymbol{x}}_{i}\left(t_{k-1}\right) \Delta t+ \\
{\left[(0.5-\alpha) \ddot{\boldsymbol{x}}_{i}\left(t_{k-1}\right)+\alpha \ddot{\boldsymbol{x}}_{i}\left(t_{k}\right)\right](\Delta t)^{2}}
\end{gathered}
$$

where $\Delta t$ denotes the discrete-time step length and $\alpha$ and $\delta(\alpha$ $\left.\geq 0.25(0.5+\delta)^{2}, \delta \geq 0.5\right)$ denote the algorithm parameters. It should be noted here that the factor $\delta$ controls the amount of artificial damping induced by the step-by-step procedure; there is no artificial damping if $\delta=0.5$. In this paper, we have $\alpha=$ $0.25, \delta=0.5$. This ensures the stability and accuracy of the Newmark- $\beta$ method. Using the fact that the current responses need to satisfy the dynamic balance of (12), we have

$$
\boldsymbol{M}_{i} \ddot{\boldsymbol{x}}_{i}\left(t_{k}\right)+\boldsymbol{C}_{i} \dot{\boldsymbol{x}}_{i}\left(t_{k}\right)+\boldsymbol{K}_{i} \boldsymbol{x}_{i}\left(t_{k}\right)=\boldsymbol{D}_{i} \boldsymbol{u}_{i}\left(t_{k}\right)
$$

Thus, combining (13)-(15), the displacements $\boldsymbol{x}_{i}\left(t_{\mathrm{k}}\right)$ can be achieved from the equivalent static equilibrium equation, i.e.,

$$
\overline{\boldsymbol{K}}_{i} \boldsymbol{x}_{i}\left(t_{k}\right)=\overline{\boldsymbol{F}}_{i}\left(t_{k}\right)
$$

where $\overline{\boldsymbol{K}}_{i}$ and $\overline{\boldsymbol{F}}_{i}$ denote the equivalent static stiffness matrix and the generalized external force vector, respectively, given as

$$
\begin{gathered}
\overline{\boldsymbol{K}}_{i}=\boldsymbol{K}_{i}+c_{0} \boldsymbol{M}_{i}+c_{1} \boldsymbol{C}_{i} \\
\overline{\boldsymbol{F}}_{i}\left(t_{k}\right)=\boldsymbol{D}_{i} \boldsymbol{u}_{i}\left(t_{k}\right)+\boldsymbol{M}_{i} \cdot\left(c_{0} \boldsymbol{x}_{i}\left(t_{k-1}\right)+c_{2} \dot{\boldsymbol{x}}_{i}\left(t_{k-1}\right)+c_{3} \ddot{\boldsymbol{x}}_{i}\left(t_{k-1}\right)\right) \\
+\boldsymbol{C}_{i} \cdot\left(c_{1} \boldsymbol{x}_{i}\left(t_{k-1}\right)+c_{4} \dot{\boldsymbol{x}}_{i}\left(t_{k-1}\right)+c_{5} \ddot{\boldsymbol{x}}_{i}\left(t_{k-1}\right)\right)
\end{gathered}
$$

where

$$
\begin{aligned}
& c_{0}=\frac{1}{\alpha(\Delta t)^{2}}, c_{1}=\frac{\delta}{\alpha \Delta t}, c_{2}=\frac{1}{\alpha \Delta t}, c_{3}=\frac{1}{2 \alpha}-1, \\
& c_{4}=\frac{\delta}{\alpha}-1, c_{5}=\frac{\Delta t}{2}\left(\frac{\delta}{\alpha}-2\right), c_{6}=\Delta t(1-\delta), c_{7}=\delta \Delta t
\end{aligned}
$$

Second, we define two types of coordinates, i.e., the internal and boundary coordinates. Then, (16) can be rewritten in a 
partitioned form, given as

$$
\left[\begin{array}{cc}
\overline{\boldsymbol{K}}_{i}^{b b} & \overline{\boldsymbol{K}}_{i}^{b s} \\
\overline{\boldsymbol{K}}_{i}^{s b} & \overline{\boldsymbol{K}}_{i}^{s s}
\end{array}\right]\left\{\begin{array}{c}
\boldsymbol{x}_{i}^{b} \\
\boldsymbol{x}_{i}^{s}
\end{array}\right\}=\left\{\begin{array}{l}
\overline{\boldsymbol{F}}_{i}^{b} \\
\overline{\boldsymbol{F}}_{i}^{s}
\end{array}\right\}
$$

where superscripts " $b$ " and "s" are the boundary and interior coordinates, respectively. Naturally, due to the symmetry of the stiffness matrix, $\overline{\boldsymbol{K}}_{i}^{b s}$ is a transpose of $\overline{\boldsymbol{K}}_{i}^{s b}$. Then, the internal variables can be determined from (19) and written as

$$
\boldsymbol{x}_{i}^{s}=\left(\overline{\boldsymbol{K}}_{i}^{s s}\right)^{-1}\left(\overline{\boldsymbol{F}}_{i}^{s}-\overline{\boldsymbol{K}}_{i}^{s b} \boldsymbol{x}_{i}^{b}\right)
$$

Substituting (20) into (19), the boundary valuables are obtained

$$
\boldsymbol{K}_{i}^{*} \boldsymbol{x}_{i}^{b}=\boldsymbol{F}_{i}^{*}
$$

where

$$
\begin{aligned}
& \boldsymbol{K}_{i}^{*}=\overline{\boldsymbol{K}}_{i}^{b b}-\overline{\boldsymbol{K}}_{i}^{b s}\left(\overline{\boldsymbol{K}}_{i}^{s s}\right)^{-1} \overline{\boldsymbol{K}}_{i}^{s b} \\
& \boldsymbol{F}_{i}^{*}=\overline{\boldsymbol{F}}_{i}^{b}-\overline{\boldsymbol{K}}_{i}^{b s}\left(\overline{\boldsymbol{K}}_{i}^{s s}\right)^{-1} \overline{\boldsymbol{F}}_{i}^{s}
\end{aligned}
$$

Equation (21) represents the condensed equilibrium equation, and the matrix $\boldsymbol{K}_{i}^{*}$ denotes the Schur complement associated with the boundary variables. After the condensation operation of all the $S$ subsystems at the 1st level grid, the remaining boundary nodes are treated as the 2 nd level grid, which can be further decomposed into several subsystems. Each subsystem at the 2 nd level grid is usually constructed by assembling several adjacent subsystems at the 1 st level grid.

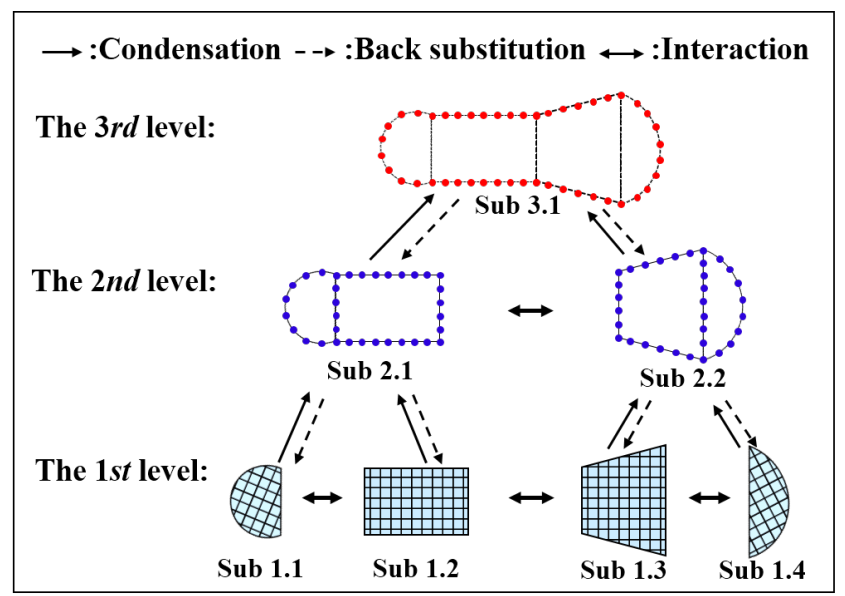

Fig. 1 Schematic of three-level physics-guided structure decomposition

To state the multilevel decomposition more intuitively, as an example, Fig. 1 gives a schematic of three-level decomposition based on the substructuring technique. As shown in Fig. 1, the notation "Sub $c . d^{\text {" }}$ " represents the $d^{\text {th }}$ subsystem of the $c^{\text {th }}$ level grid. The overall system is decomposed into four subsystems that are regarded as the 1st level. After condensation operation (i.e., the solid arrow in Fig. 1) by (19), (21) and (22), each subsystem at the 1st level can be assembled into the 2nd level. Then, the system model at the 2nd level is further decomposed into two small subsystems that are composed of several associated adjacent subsystems from the 1 st level grid. In general, the $j^{\text {th }}$ subsystem model at the $(l+1)^{\text {th }}$ level $(l \geq 1)$ can be formulated by condensing and assembling the corresponding $S_{l}$ adjacent subsystems from the $l^{\text {th }}$ level, given as

$$
\boldsymbol{K}_{j}^{(l+1)} \cdot \bigwedge_{i=1}^{S_{l}} \boldsymbol{x}_{i}^{(l)}=\boldsymbol{F}_{j}^{(l+1)}
$$

where the operator $\bigwedge_{i=1}^{S_{l}}$ represents the assembly over the $S_{l}$ subsystems of the $l^{\text {th }}$ level grid, and

$$
\begin{aligned}
& \boldsymbol{K}_{j}^{(l+1)}=\bigwedge_{i=1}^{S_{l}} \boldsymbol{K}_{i}^{(l)}, \boldsymbol{K}_{i}^{(l)}=\left[\overline{\boldsymbol{K}}_{i}^{b b}-\overline{\boldsymbol{K}}_{i}^{b s}\left(\overline{\boldsymbol{K}}_{i}^{s s}\right)^{-1} \overline{\boldsymbol{K}}_{i}^{s b}\right]^{(l)} \\
& \boldsymbol{F}_{j}^{(l+1)}=\bigwedge_{i=1}^{S_{l}} \boldsymbol{F}_{i}^{(l)}, \boldsymbol{F}_{i}^{(l)}=\left\{\overline{\boldsymbol{F}}_{i}^{b}-\overline{\boldsymbol{K}}_{i}^{b s}\left(\overline{\boldsymbol{K}}_{i}^{s s}\right)^{-1} \overline{\boldsymbol{F}}_{i}^{s}\right\}^{(l)}
\end{aligned}
$$

Hence, in a similar way, the multilevel decomposition will finally reach the top level grid.

Finally, all the displacements at the top level are regarded as the internal variables, which can be directly computed by

$$
\boldsymbol{x}^{(t o p)}=\left[\boldsymbol{K}^{(t o p)}\right]^{-1} \boldsymbol{F}^{(t o p)}
$$

The displacements of each subsystem at other levels can be calculated from the higher to the lower levels by a so-called back substitution operation (i.e., the dashed arrow in Fig. 1).

$$
\begin{aligned}
& \boldsymbol{x}_{j}^{b(l)}=\left\{\left(\boldsymbol{x}_{i}^{b(l+1)}\right)^{\mathrm{T}},\left(\boldsymbol{x}_{i}^{s(l+1)}\right)^{\mathrm{T}}\right\}_{j}^{\mathrm{T}} \\
& \boldsymbol{x}_{j}^{s(l)}=\left\{\left(\overline{\boldsymbol{K}}_{j}^{s s}\right)^{-1}\left(\overline{\boldsymbol{F}}_{j}^{s}-\overline{\boldsymbol{K}}_{j}^{s b} \boldsymbol{x}_{j}^{b}\right)^{(l)}, j=1,2, \cdots, S_{l}\right.
\end{aligned}
$$

Subsequently, the acceleration and velocity variables of each subsystem at time $t_{k}$ can be derived as

$$
\begin{aligned}
& \ddot{\boldsymbol{x}}_{i}\left(t_{k}\right)=c_{0}\left(\boldsymbol{x}_{i}\left(t_{k}\right)-\boldsymbol{x}_{i}\left(t_{k-1}\right)\right)-c_{2} \dot{\boldsymbol{x}}_{i}\left(t_{k-1}\right)-c_{3} \ddot{\boldsymbol{x}}_{i}\left(t_{k-1}\right) \\
& \dot{\boldsymbol{x}}_{i}\left(t_{k}\right)=\dot{\boldsymbol{x}}_{i}\left(t_{k-1}\right)+c_{6} \ddot{\boldsymbol{x}}_{i}\left(t_{k-1}\right)+c_{7} \ddot{\boldsymbol{x}}_{i}\left(t_{k}\right)
\end{aligned}
$$

So far, based on the substructuring technique, the entire system has been decomposed into a series of multilevel subsystems.

\section{B. Prediction Models in Different Levels}

In the MPC framework, a model of the controlled system is used to predict future responses. The efficiency of the calculated control actions of an MPC depends heavily upon the prediction model of system [28]. Based on the aforementioned multilevel decomposition mode, prediction models for different local subsystems can be established at different levels.

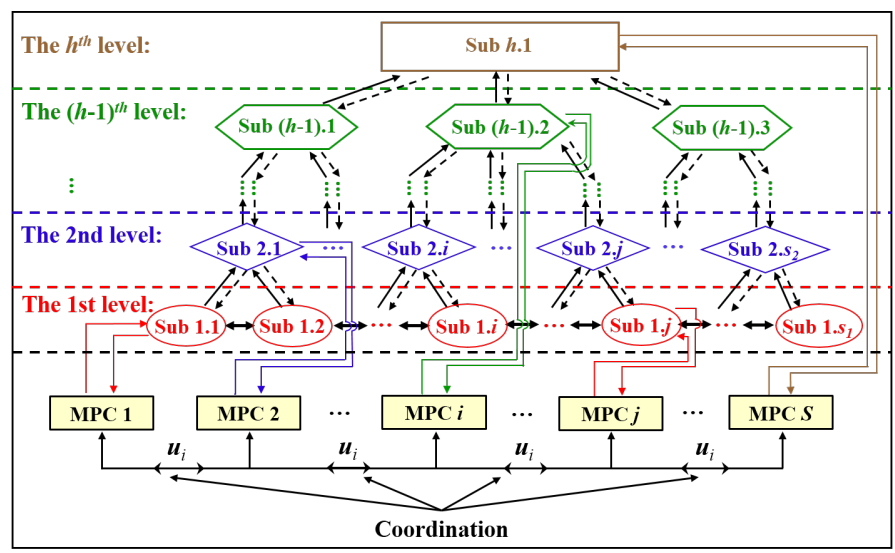

Fig. 2 The proposed multilevel DMPC architecture

Fig. 2 presents a schematic of the proposed multilevel DMPC framework. As shown in Fig. 2, an $h$-level structural system can generally be decomposed based on the topological shape, actuator arrangement, and calculation scale of the system. Along with the system decomposition, all the actuators embedded as partial members of the structure are divided into 
several subsets. Each actuator subset should be controlled by a local MPC controller. For instance, the local MPC controller for the first actuator subset can be designed based on the prediction model of not only the subsystem of Sub 1.1 in the 1st level but also the corresponding subsystem of Sub 2.1 in the 2nd level when the nodes of actuator elements are treated as the boundary nodes. In fact, the control law of a distributed MPC controller can be designed based on the subsystem model at any level grid, as long as the model involves all the actuator element nodes. Furthermore, because more structural information, involving the equivalent static stiffness and external force, is condensed in the higher-level subsystems, a better control effect, in theory, will be achieved when a model at a higher level is used for the controller design.

Remark 1: The proposed DMPC based on this decomposition mode can naturally degenerate into a centralized MPC (CMPC) if the overall structure is regarded as one substructure; and the substructuring technique can still be used to reduce the scale of the prediction model for controller design.

\section{CoORdinated Distributed MPC For SHAPE CONTROL WITH INPUT SATURATION}

In this section, a coordinated multilevel DMPC framework for shape control with input saturation is introduced.

\section{A. Discretization by the Explicit Newmark- $\beta$ Method}

To solve the DMPC problem, the subsystem prediction model (5) should be discretized in the time domain; then the state evolution can be predicted by the discrete convolution integral [30]. However, this discretization approach involves considerable off-line computation of matrix exponentials, especially for a large-scale system [31]. Thus, to improve the computational efficiency, an explicit Newmark- $\beta$ algorithm is applied to discretize the subsystem (3).

The prediction time horizon $T$ is divided into $N$ time slots with equal step length $\eta$. The dynamic balance equation at time $t_{k}$ can be written as

$$
\hat{\boldsymbol{M}}_{\mathrm{i}} \ddot{\boldsymbol{x}}_{i}\left(t_{k}\right)+\hat{\boldsymbol{C}}_{i} \dot{\boldsymbol{x}}_{i}\left(t_{k}\right)+\hat{\boldsymbol{K}}_{i} \boldsymbol{x}_{i}\left(t_{k}\right)=\boldsymbol{D}_{i i} \boldsymbol{u}_{i}\left(t_{k}\right)+\sum_{j \neq i}^{S} \boldsymbol{D}_{i j} \boldsymbol{u}_{j}\left(t_{k}\right)
$$

Combining (28) and the aforementioned basic assumptions for the velocity and displacement in the Newmark- $\beta$ algorithm (i.e., (13) and (14), respectively), the dynamic responses $\boldsymbol{v}_{i}\left(t_{k}\right)(\boldsymbol{v}=$ $\left.\left\{\boldsymbol{x}^{\mathrm{T}}, \dot{\boldsymbol{x}}^{\mathrm{T}}, \ddot{\boldsymbol{x}}^{\mathrm{T}}\right\}^{\mathrm{T}}\right)$ can be derived in an explicit form

$$
\boldsymbol{v}_{i}\left(t_{k}\right)=\boldsymbol{h}_{i} \boldsymbol{v}_{i}\left(t_{k-1}\right)+\boldsymbol{w}_{i i} \boldsymbol{u}_{i}\left(t_{k}\right)+\sum_{j \neq i}^{S} \boldsymbol{w}_{i j} \boldsymbol{u}_{j}\left(t_{k}\right)
$$

where

$$
\boldsymbol{h}_{i}=\left[\begin{array}{lll}
\boldsymbol{\Psi}_{11} & \boldsymbol{\Psi}_{12} & \boldsymbol{\Psi}_{13} \\
\boldsymbol{\Psi}_{21} & \boldsymbol{\Psi}_{22} & \boldsymbol{\Psi}_{23} \\
\boldsymbol{\Psi}_{31} & \boldsymbol{\Psi}_{32} & \boldsymbol{\Psi}_{33}
\end{array}\right], \boldsymbol{w}_{i i}=\left[\begin{array}{c}
\boldsymbol{\Gamma}_{i}^{-1} \boldsymbol{D}_{i i} \\
b_{1} \boldsymbol{\Gamma}_{i}^{-1} \boldsymbol{D}_{i i} \\
b_{4} \boldsymbol{\Gamma}_{i}^{-1} \boldsymbol{D}_{i i}
\end{array}\right], \boldsymbol{w}_{i j}=\left[\begin{array}{c}
\boldsymbol{\Gamma}_{i}^{-1} \boldsymbol{D}_{i j} \\
b_{1} \boldsymbol{\Gamma}_{i}^{-1} \boldsymbol{D}_{i j} \\
b_{4} \boldsymbol{\Gamma}_{i}^{-1} \boldsymbol{D}_{i j}
\end{array}\right]
$$

where $\boldsymbol{\Gamma}_{i}=b_{4} \hat{\boldsymbol{M}}_{i}+b_{1} \hat{\boldsymbol{C}}_{i}+\hat{\boldsymbol{K}}_{i}$, and the specific expression for $b_{\kappa}$ $(\kappa=1,2,3,4,5,6)$ and $\Psi_{\mu v}(\mu, v=1,2,3)$ can be found in [31]. Performing an iterative calculation of the recurrence formula (29), all the future dynamic responses can be expressed by the predictive initial state and control inputs, given as

$$
\boldsymbol{v}_{i}\left(t_{k}\right)=\hat{\boldsymbol{H}}_{i}^{k} \boldsymbol{z}_{i}\left(t_{0}\right)+\sum_{l=0}^{k} \hat{\boldsymbol{W}}_{i i}^{k, l} \boldsymbol{u}_{i}\left(t_{l}\right)+\sum_{j \neq i}^{S}\left\{\sum_{l=0}^{k} \hat{\boldsymbol{W}}_{i j}^{k, l} \boldsymbol{u}_{j}\left(t_{l}\right)\right\}
$$

where the subscript " $i$ " denotes the $i^{\text {th }}$ subsystem, superscripts " $k$ " and " $l "$ denote the predictive time step, and

$$
\begin{gathered}
\hat{\boldsymbol{H}}_{i}^{k}=\boldsymbol{h}_{i}^{k} \boldsymbol{g}_{i 1}, \boldsymbol{W}_{i i}^{k, l}=\left\{\begin{array}{l}
\boldsymbol{h}_{i}^{k} \boldsymbol{g}_{i 2}, \quad l=0 \\
\boldsymbol{h}_{i}^{k-l} \boldsymbol{w}_{i i}, 1 \leq l \leq k
\end{array}, \hat{\boldsymbol{W}}_{i j}^{k, l}=\left\{\begin{array}{l}
\boldsymbol{h}_{i}^{k} \boldsymbol{g}_{i 3}, \quad l=0 \\
\boldsymbol{h}_{i}^{k-l} \boldsymbol{w}_{i j}, 1 \leq l \leq k
\end{array}\right.\right. \\
\boldsymbol{g}_{i 1}=\left[\begin{array}{cc}
\boldsymbol{I}_{i} & \mathbf{0} \\
\mathbf{0} & \boldsymbol{I}_{i} \\
-\hat{\boldsymbol{M}}_{i}^{-1} \boldsymbol{K}_{i} & -\hat{\boldsymbol{M}}_{i}^{-1} \boldsymbol{C}_{i}
\end{array}\right], \boldsymbol{g}_{i 2}=\left[\begin{array}{c}
\mathbf{0} \\
\mathbf{0} \\
\hat{\boldsymbol{M}}_{i}^{-1} \boldsymbol{D}_{i i}
\end{array}\right], \boldsymbol{g}_{i 3}=\left[\begin{array}{c}
\mathbf{0} \\
\mathbf{0} \\
\hat{\boldsymbol{M}}_{i}^{-1} \boldsymbol{D}_{i j}
\end{array}\right]
\end{gathered}
$$

\section{B. Coordination Mechanism among Local Controllers}

To improve the systemwide control performance of DMPC, the local MPC controllers should cooperate with each other to achieve a systemwide objective. Thus, the local cost function $\varphi_{i}$ should be replaced by a cost function, which can measure the impact of local control actions on the whole system. Drawing on the concept of cooperative DMPC proposed by Venkat et al. [32], [33], a simple choice for such a cost function is to combine all the local cost functions in the following way:

$$
\Phi=\sum_{i=1}^{S} \omega_{i} \varphi_{i}, \omega_{i} \geq 0, \sum_{i=1}^{S} \omega_{i}=1
$$

In this work, we choose $\omega_{i}=1 / S$.

Subsequently, an iterative implementation strategy is used to minimize the aforementioned systemwide cost function $\Phi$ of (33). In this strategy, each controller optimizes its own set of inputs assuming that the rest of the inputs for other interacting subsystems are fixed to the last accepted values. Following this step, as shown in Fig. 2, the controllers share their optimal solutions of the future inputs (i.e., the process of coordination between each local controller). If the preset convergence condition is satisfied, each local controller will send its final optimal inputs $\boldsymbol{u}_{i}^{p}$ to the corresponding actuators; if not, it should go to the next iteration $p+1$, and the current inputs will be updated by a convex combination of the most recent input trajectory and that at the last iteration, i.e.,

$$
\boldsymbol{u}_{i}^{p+1}=\omega_{i} \boldsymbol{u}_{i}^{p}+\left(1-\omega_{i}\right) \boldsymbol{u}_{i}^{p-1}
$$

For the $p^{\text {th }}$ iteration at time $t_{k}$, the coordinated DMPC of each subsystem is described as a constrained quadratic programming (QP) problem in the discrete-time domain, that is

Find: $\quad \boldsymbol{u}_{i}^{p}\left(t_{k}\right) \in \underset{U^{p}}{\arg \min } \varphi_{i}\left(\boldsymbol{Y}_{i}^{p}, \boldsymbol{U}_{i}^{p}, \boldsymbol{U}_{j \neq i}^{p-1} ; \boldsymbol{z}_{i}\left(t_{k}\right)\right)$

Subject to: $\quad \boldsymbol{y}_{i}^{p}(k+q \mid k)=\overline{\boldsymbol{C}}_{i} \boldsymbol{v}_{i}^{p}(k+q \mid k)$

$$
\boldsymbol{\mu}_{\text {min }} \leq \boldsymbol{u}_{i}^{p}(k+q \mid k) \leq \boldsymbol{\mu}_{\max }
$$

With:

$$
\begin{aligned}
& \varphi_{i}\left(\boldsymbol{Y}_{i}^{p}, \boldsymbol{U}_{i}^{p}, \boldsymbol{U}_{j \neq i}^{p-1} ; \boldsymbol{z}_{i}\left(t_{k}\right)\right) \\
& =\frac{1}{2} \sum_{q=0}^{N}\left(\left\|\boldsymbol{y}_{i}^{p}(k+q \mid k)-\boldsymbol{y}_{i d}\right\|_{\boldsymbol{Q}_{i}}^{2}+\left\|\boldsymbol{u}_{i}^{p}(k+q \mid k)\right\|_{\boldsymbol{R}_{i}}^{2}\right)
\end{aligned}
$$

where $\boldsymbol{Y}_{i}^{p}=\left[\boldsymbol{y}_{i}^{p}(k+1 \mid k)^{\mathrm{T}}, \cdots, \boldsymbol{y}_{i}^{p}(k+N \mid k)^{\mathrm{T}}\right]^{\mathrm{T}}, \boldsymbol{U}_{i}^{p}=\left[\boldsymbol{u}_{i}^{p}(k \mid k\right.$ )$\left.^{\mathrm{T}}, \cdots, \boldsymbol{u}_{i}^{p}(k+N \mid k)^{\mathrm{T}}\right]^{\mathrm{T}}, \boldsymbol{U}_{i}^{p-1}=\left[\boldsymbol{u}_{i}^{p-1}(k \mid k)^{\mathrm{T}}, \ldots, \boldsymbol{u}_{i}^{p-1}(k+N \mid k)^{\mathrm{T}}\right]^{\mathrm{T}} ;$ $y_{i}^{p}$ represents the output values at iteration $p$; and the double 
index notation $(k+q \mid k)$ represents a prediction for $q(1 \leq q \leq N)$ steps ahead from time $t_{k}$.

Herein, it should be noted that the system sampling interval may be insufficient for a coordination-based iterative method to reach convergence, especially for large-scale systems. Thus, in such cases, the iteration has to be terminated prior to convergence of optimal input trajectories. The results of the last iteration will be used as an approximate distributed control law.

Remark 2: Consider the above cost function $\Phi$ positive definite quadratic and let the solution domain for the problem of (35)-(38) be convex and compact. Assume that the solution after $p$ iterates is $\left(\boldsymbol{u}_{1}^{p}, \ldots, \boldsymbol{u}_{S}^{p}\right)$ and the corresponding cost function value is $\Phi\left(\boldsymbol{u}_{1}^{p}, \ldots, \boldsymbol{u}_{S}^{p} ; \boldsymbol{z}\left(t_{k}\right)\right)$; let the optimal solution be $\left(\boldsymbol{u}_{1}^{*}, \ldots, \boldsymbol{u}_{S}^{*}\right)$ with a relevant cost function $\Phi\left(\boldsymbol{u}_{1}^{*}, \ldots, \boldsymbol{u}_{S}^{*} ; \boldsymbol{z}\left(t_{k}\right)\right)$. The input trajectory at convergence satisfies

$$
\begin{aligned}
\lim _{p \rightarrow \infty} \Phi\left(\boldsymbol{u}_{1}^{p}, \boldsymbol{u}_{2}^{p}, \cdots, \boldsymbol{u}_{S}^{p} ; \boldsymbol{z}_{i}\left(t_{k}\right)\right) & =\Phi\left(\boldsymbol{u}_{1}^{*}, \boldsymbol{u}_{2}^{*}, \cdots, \boldsymbol{u}_{S}^{*} ; \boldsymbol{z}_{i}\left(t_{k}\right)\right) \\
\lim _{p \rightarrow \infty}\left(\boldsymbol{u}_{1}^{p}, \boldsymbol{u}_{2}^{p}, \cdots, \boldsymbol{u}_{S}^{p}\right) & =\left(\boldsymbol{u}_{1}^{*}, \boldsymbol{u}_{2}^{*}, \cdots, \boldsymbol{u}_{S}^{*}\right)
\end{aligned}
$$

A proof can be found in [32, p. 1024].

\section{Solution of the DMPC Method with Input Saturation}

In this subsection, the aforementioned constrained QP problem (35)-(38) is transformed into an LCP problem based on the parametric variational principle [34].

First, for the equality constraint of (36), substituting (31) into (36), yields

$$
\begin{gathered}
\boldsymbol{y}_{i}^{p}(k+q \mid k)=\overline{\boldsymbol{C}}_{i} \hat{\boldsymbol{H}}_{i}^{q} \boldsymbol{z}_{i}\left(t_{k}\right)+\sum_{l=0}^{q} \overline{\boldsymbol{C}}_{i} \hat{\boldsymbol{W}}_{i i}^{q, l} \boldsymbol{u}_{i}^{p}\left(t_{l}\right) \\
+\sum_{j \neq i}^{S}\left\{\sum_{l=0}^{q} \overline{\boldsymbol{C}}_{i} \hat{\boldsymbol{W}}_{i j}^{q, l} \boldsymbol{u}_{j}^{p-1}\left(t_{l}\right)\right\}
\end{gathered}
$$

Equation (40) can be rewritten in a matrix form as

$$
\boldsymbol{Y}_{i}^{p}=\boldsymbol{H}_{i} \boldsymbol{z}_{i}\left(t_{k}\right)+\boldsymbol{W}_{i i} \boldsymbol{U}_{i}^{p}+\sum_{j \neq i}^{S} \boldsymbol{W}_{i j} \boldsymbol{U}_{j}^{p-1}
$$

Second, the inequality constraint (37) can be addressed by transforming the original problem into an $\mathrm{LCP}$ in a very concise and clear way. First, the inequality constraint (37) is transformed into an equality constraint by introducing variables $\overline{\boldsymbol{\alpha}}_{i} \geq 0$ and $\underline{\boldsymbol{\alpha}}_{i}$, which can be expressed in matrix form

$$
\left\{\begin{array}{l}
\boldsymbol{U}_{i}^{p}-\overline{\boldsymbol{E}}_{i} \boldsymbol{\mu}_{\max }+\overline{\boldsymbol{\alpha}}_{i}=\mathbf{0} \\
\overline{\boldsymbol{E}}_{i} \boldsymbol{\mu}_{\min }-\boldsymbol{U}_{i}^{p}+\underline{\boldsymbol{\alpha}}_{i}=\mathbf{0}
\end{array}\right.
$$

where $\overline{\boldsymbol{E}}_{i}$ is a matrix for dimension expansion. Then, based on the theory of the parametric variational principle, an expanded cost function $\varphi_{i}^{e}(38)$ is obtained, which can be defined as

$$
\begin{aligned}
\varphi_{i}^{e} & =\frac{1}{2}\left(\left\|\boldsymbol{Y}_{i}^{p}-\boldsymbol{Y}_{i d}\right\|_{\hat{\underline{Q}}_{i}}^{2}+\left\|\boldsymbol{U}_{i}^{p}\right\|_{\hat{\boldsymbol{R}}_{i}}^{2}\right) \\
& +\overline{\boldsymbol{\lambda}}_{i}^{T}\left(\boldsymbol{U}_{i}^{p}-\overline{\boldsymbol{E}}_{i} \boldsymbol{\mu}_{\max }+\overline{\boldsymbol{\alpha}}_{i}\right)+\underline{\boldsymbol{\lambda}}_{i}^{T}\left(\overline{\boldsymbol{E}}_{i} \boldsymbol{\mu}_{\min }-\boldsymbol{U}_{i}^{p}+\underline{\boldsymbol{\alpha}}_{i}\right)
\end{aligned}
$$

where $\widehat{\boldsymbol{Q}}_{i}=\operatorname{diag}\left\{\boldsymbol{Q}_{i}(1), \boldsymbol{Q}_{i}(2), \ldots, \boldsymbol{Q}_{i}(N-1), \overline{\boldsymbol{Q}}_{i}(N)\right\}$ and $\widehat{\boldsymbol{R}}_{i}=$ $\operatorname{diag}\left\{\boldsymbol{R}_{i}(0), \boldsymbol{R}_{i}(1), \ldots, \boldsymbol{R}_{i}(N)\right\}, \overline{\boldsymbol{Q}}_{i}$ is an appropriately chosen terminal penalty; $\bar{\lambda}_{i}$ and $\underline{\lambda}_{i}$ are the Lagrange multipliers. Taking into account (41), the output variables $\boldsymbol{Y}_{i}^{p}$ in (43) can be eliminated. Thus, the original constrained QP problem can be changed into an unconstrained optimization problem, given as

$$
\boldsymbol{u}_{i}^{p}\left(t_{k}\right) \in \underset{\boldsymbol{U}_{i}^{p}}{\arg \min } \varphi_{i}^{e}:=\varphi_{i}^{e}\left(\boldsymbol{U}_{i}^{p}, \boldsymbol{U}_{j \neq i}^{p-1} ; \bar{\lambda}_{i}, \underline{\lambda}_{i}, \boldsymbol{z}_{i}\left(t_{k}\right)\right)
$$

By performing the variation operation with respect to the input trajectory $\boldsymbol{U}_{i}^{p}$, that is,

$$
\partial \varphi_{i}^{e} / \partial \boldsymbol{U}_{i}^{p}=\mathbf{0}
$$

the suboptimal input trajectory $\boldsymbol{U}_{i}^{p}$ can be derived in an explicit form, which only depends on $\bar{\lambda}_{i}$ and $\underline{\lambda}_{i}$, given as

$$
\boldsymbol{U}_{i}^{p}=\boldsymbol{G}_{i 1}\left(\bar{\lambda}_{i}^{T}-\underline{\lambda}_{i}^{T}\right)+\boldsymbol{G}_{i 2} \boldsymbol{H}_{i} \boldsymbol{z}_{i}+\boldsymbol{G}_{i 3}
$$

where

$$
\begin{aligned}
& \boldsymbol{G}_{i 1}=-\left(\boldsymbol{W}_{i i}^{\mathrm{T}} \hat{\boldsymbol{Q}}_{i} \boldsymbol{W}_{i i}+\hat{\boldsymbol{R}}_{i}\right)^{-1}, \boldsymbol{G}_{i 2}=\boldsymbol{G}_{i 1} \boldsymbol{W}_{i i}^{\mathrm{T}} \hat{\boldsymbol{Q}}_{i} \\
& \boldsymbol{G}_{i 3}=\boldsymbol{G}_{i 2}\left(\sum_{j \neq i}^{S} \boldsymbol{W}_{i j} \boldsymbol{U}_{j}^{p-1}-\boldsymbol{Y}_{i d}\right)
\end{aligned}
$$

Finally, substituting (46) into (42), the constraint conditions can be rewritten as

$$
\left\{\begin{array}{l}
\boldsymbol{G}_{i 2} \boldsymbol{H}_{\mathrm{i}} z_{\mathrm{i}}+\boldsymbol{G}_{i 3}-\overline{\boldsymbol{E}}_{i} \boldsymbol{\mu}_{\max } \\
\overline{\boldsymbol{E}}_{i} \boldsymbol{\mu}_{\min }-\boldsymbol{G}_{i 2} \boldsymbol{H}_{\mathrm{i}} z_{i}-\boldsymbol{G}_{i 3}
\end{array}\right\}+\left[\begin{array}{cc}
\boldsymbol{G}_{i 1} & -\boldsymbol{G}_{i 1} \\
-\boldsymbol{G}_{i 1} & \boldsymbol{G}_{i 1}
\end{array}\right]\left\{\begin{array}{l}
\bar{\lambda}_{i} \\
\underline{\lambda}_{i}
\end{array}\right\}+\left\{\begin{array}{l}
\overline{\boldsymbol{\alpha}}_{i} \\
\underline{\boldsymbol{\alpha}}_{i}
\end{array}\right\}=\mathbf{0}
$$

Thus far, the original DMPC problem with input saturation can be transformed into a series of LCP problems. $\bar{\lambda}_{i}$ and $\underline{\lambda}_{i}$ can then be obtained by solving these LCP problems as follows:

$$
\left\{\begin{array}{l}
\boldsymbol{\zeta}_{i}+\boldsymbol{T}_{i} \boldsymbol{v}_{i}+\boldsymbol{\gamma}_{i}=\mathbf{0} \\
\boldsymbol{v}_{i} \geq \mathbf{0}, \boldsymbol{\gamma}_{i} \geq \mathbf{0}, \boldsymbol{v}_{i}^{\mathrm{T}} \gamma_{i}=0
\end{array}\right.
$$

where

$$
\begin{aligned}
& \boldsymbol{\zeta}_{i}=\left\{\begin{array}{l}
\boldsymbol{G}_{i 2} \mathbf{H}_{i} \mathbf{z}_{i}+\boldsymbol{G}_{i 3}-\overline{\boldsymbol{E}}_{i} \boldsymbol{\mu}_{\max } \\
\overline{\boldsymbol{E}}_{i} \boldsymbol{\mu}_{\min }-\boldsymbol{G}_{i 2} \mathbf{H}_{i} \mathbf{z}_{i}-\boldsymbol{G}_{i 3}
\end{array}\right\} \\
& \boldsymbol{T}_{i}=\left[\begin{array}{cc}
\boldsymbol{G}_{i 1} & -\boldsymbol{G}_{i 1} \\
-\boldsymbol{G}_{i 1} & \boldsymbol{G}_{i 1}
\end{array}\right], \boldsymbol{v}_{i}=\left\{\begin{array}{l}
\overline{\boldsymbol{\lambda}}_{i} \\
\underline{\boldsymbol{\lambda}}_{i}
\end{array}\right\}, \gamma_{i}=\left\{\begin{array}{l}
\overline{\boldsymbol{\alpha}}_{i} \\
\underline{\boldsymbol{\alpha}}_{i}
\end{array}\right\}
\end{aligned}
$$

The LCP can be solved by many mature approaches. Herein, Lemke's algorithm [35] is used to solve the standard LCP. TABLE I

THE DMPC ALGORITHM FRAMEWORK FOR STRUCTURAL SHAPE CONTROL

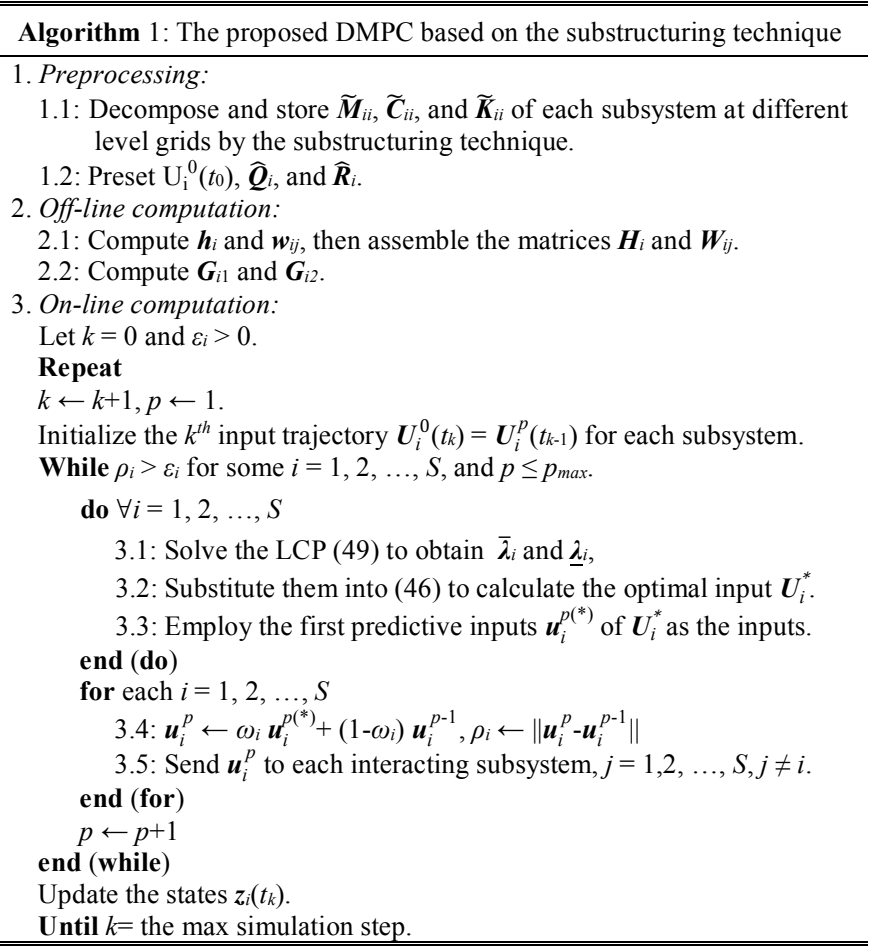


The implementation steps of the proposed DMPC algorithm for structural shape control are summarized in Table I. In addition, all the parameters in the above theoretical formulas are listed in Table VII of Appendix B.

Remark 3: One merit of the proposed DMPC method is that the input saturation constraints can be satisfied directly just by solving the LCP, rather than by adjusting the control parameters such as the weighting matrices to ensure that the actuators work under their physical limits indirectly.

Remark 4 (stability): If $\left(\boldsymbol{A}_{i i}, \overline{\boldsymbol{C}}_{i}\right)$ is detectable, $\boldsymbol{Q}_{i} \geq 0$ and $\boldsymbol{R}_{i}>$ $0, \forall i \in\{1, S\}$, the closed-loop system with the control input trajectory solved by the proposed Algorithm 1 can be proven to be stable at the origin. A proof by Lyapunov stability theory [36] is given in Appendix A.

\section{EXPERIMENTAL EVALUATION}

In this section, an experiment on the shape control of a smart antenna reflector is investigated to illustrate the performance of the proposed DMPC controller. The control problem and some pretreatment including the experiment platform, the reflector model, and the system decomposition are briefly introduced in subsection A. The feasibility, effectiveness, flexibility, and fault tolerance of the proposed algorithm are discussed in subsection $\mathrm{B}$. It should be noted that all computations are performed in MATLAB (R2016b) on a personal computer with an Intel(R) Core(TM) i7-7600 CPU (2.80 GHz) processor and 8 GB RAM, running on Windows 1064 bit.

\section{A. Experiment Introduction and Physics-guided System Decomposition of the Smart Antenna Reflector}

A planar hexagonal reflector with an inscribed circle diameter of $0.654 \mathrm{~m}$ is selected as the research object. The host structure is manufactured by composite with eight layers of carbon fibers; each layer is $1.25 \times 10^{-4} \mathrm{~m}$ thick. The ply orientations are $\left(0^{\circ}, 45^{\circ},-45^{\circ}, 90^{\circ}\right)$, where the superscript "o" denotes the degree of angle. Drawing on the concept of the reflector structure reported in [3], thirty active PZT actuators are embedded on the ribs at the back of the reflector, as shown in Fig. 3. The details about the rib design, actuator installation, and physical constraints of the structure can be found in [3]. The material properties for both the composite and PZT actuator elements are listed in Table II. The active structural shape control problem in this experiment can be described such that the aim is to find the optimal control inputs (i.e., the voltages of PZT actuators) by the proposed DMPC algorithm to make the displacement field of the reflector surface a parabola with a focal length of $100 \mathrm{~m}$.

TABLE II

PROPERTIES OF BOTH THE COMPOSITE AND PZT ACTUATOR ELEMENTS

\begin{tabular}{ccc}
\hline \hline Parameters & Composite elements & PZT elements \\
\hline Young's modulus $(\mathrm{GPa})$ & $E_{1}=263, E_{2}=E_{3}=11$ & $E^{\mathrm{e}}=26.53$ \\
Shear modulus $(\mathrm{GPa})$ & $G_{12}=G_{13}=5.28, G_{23}=4.23$ & $/$ \\
Poison's ratio & $\mu=0.3$ & $/$ \\
Density $\left(\mathrm{kg} / \mathrm{m}^{3}\right)$ & $\rho=1800$ & $\rho^{e}=8000$ \\
Area of cross section $\left(\mathrm{mm}^{2}\right)$ & $/$ & $A^{\mathrm{e}}=113.10$ \\
Actuator length $(\mathrm{m})$ & $/$ & $l^{\mathrm{e}}=0.10$ \\
PZT stress coefficient $(\mathrm{pm} / \mathrm{V})$ & $/$ & $\kappa_{33}=635$ \\
The item $A^{\mathrm{e}} /\left(l^{\mathrm{e}} \omega_{33}\right)$ & $/$ & 12 \\
Actuator voltage range $(\mathrm{V})$ & $/$ & $0-150$ \\
\hline \hline
\end{tabular}

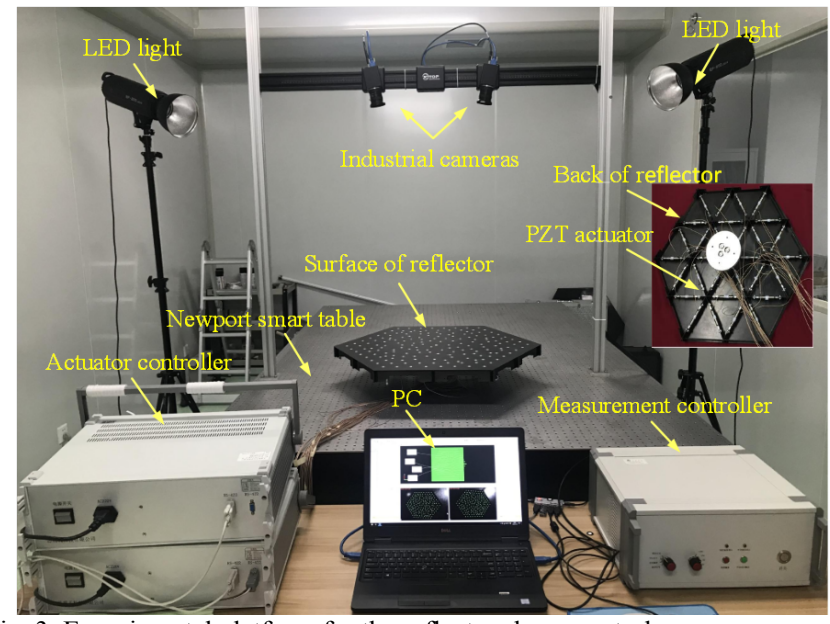

Fig. 3 Experimental platform for the reflector shape control

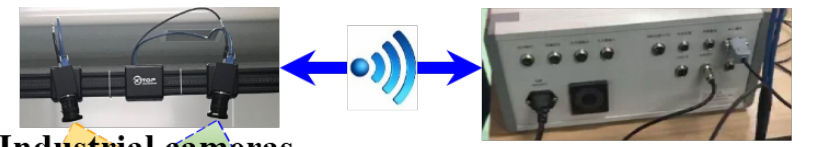

Industrial cámęras
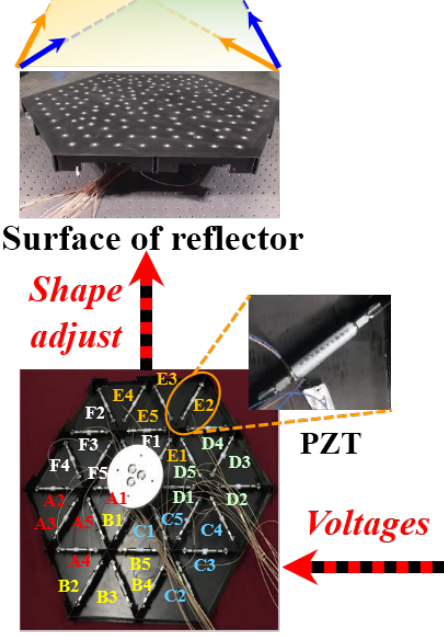

Back of reflector

Fig. 4 Schematic of the data flow for the control system

Fig. 3 gives the schematic of the experimental platform for reflector shape control. A digital image correlation (DIC) photogrammetry system with an accuracy of $1 \times 10^{-5} \mathrm{~m}$ is used to measure the special position of the reflector surface in real time. This measurement system is composed of two industrial cameras, one measurement controller and two light-emitting diode (LED) lights. When an image capture command is received, the current surface deformation is captured by the cameras, and the coordinates of the observation points, i.e., the white dots pasted on the reflector surface, are sent back to the measurement controller. Then, an Ethernet cable is used for communicating between the measurement controller and the PC. Based on the feedback states of the system, the suboptimal control voltages of each subsystem are solved by the proposed DMPC method in MATLAB. Subsequently, the voltage signals are amplified and loaded into each actuator subset by the actuator controller. In addition, the reflector is fixed on a Newport Smart Table, which is composed of four smart dampers and one breadboard with $0.1 \mathrm{~mm}$ surface flatness to 
reduce the impact of external disturbance. A schematic view of the data flow for the control system is given in Fig. 4.

Based on the substructuring technique, a three-level system decomposition is presented as shown in Fig. 5. As the overall structural system is divided into 6 subsystems, all 30 PZT actuators are equally divided into 6 subsets at the same time; each actuator subset is controlled by different local MPC controllers with information interactions. Benefiting from this multilevel system decomposition mode, different MPC controllers can be designed flexibly at different levels.

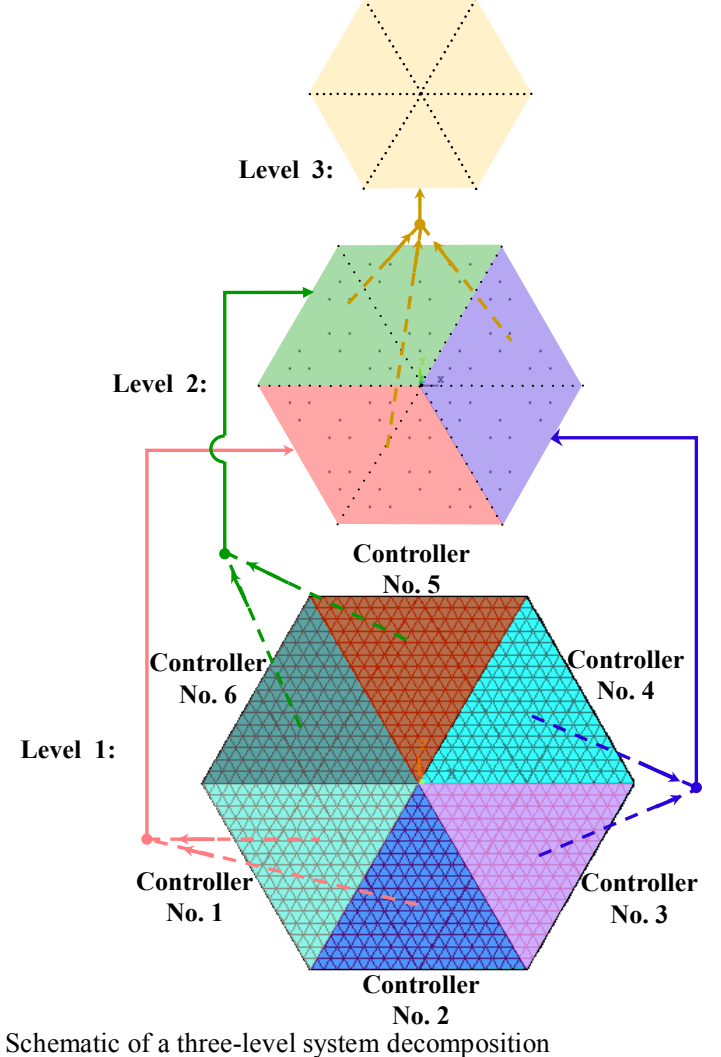

Fig. 5 Schematic of a three-level system decomposition

\section{B. Shape Control of the Antenna Reflector using the Proposed DMPC Method}

The experiment on shape control of the antenna reflector by the proposed multilevel DMPC will be discussed in four parts, including the model verification, feasibility and effectiveness, flexibility, and fault tolerance of the proposed algorithm.

1) model verification:

To illustrate the correctness of the numerical model for the reflector system, certain groups of constant voltages are applied to the PZT actuators; and the numerical results involving the displacement field and its root mean square (RMS.) value of the reflector surface are compared with those in the experiment.

As presented in Fig. 6, the displacement field by numerical simulation under loading at a $70 \mathrm{~V}$ control voltage is very close to that in the experiment. The displacement RMS values are $135.12 \mu \mathrm{m}$ and $139.53 \mu \mathrm{m}$, respectively. Without loss of generality, the maximum (Max.) and RMS of the displacements at different voltages of $30 \mathrm{~V}, 70 \mathrm{~V}$, and $140 \mathrm{~V}$ are further listed in Table III. Compared with the experimental results, the errors for the RMS of displacement by numerical simulation are
$4.68 \%, 3.16 \%$, and $3.02 \%$, respectively. Thus far, all the comparison results illustrate that the numerical model of the reflector for a DMPC controller is available with enough accuracy.

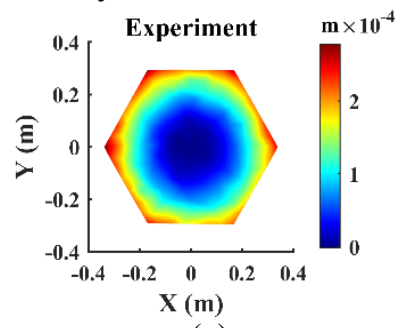

(a) (b)

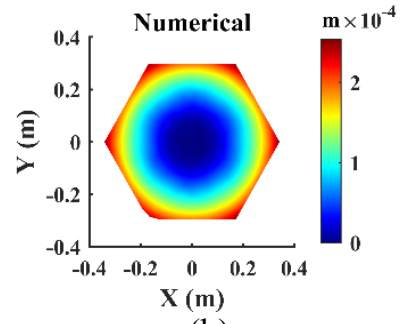

Fig. 6 Comparisons of the displacement fields at the control voltage of $70 \mathrm{~V}$ : (a) The experimental results. (b) The numerical results.

TABLE III

THE MAX. AND RMS OF THE DISPLACEMENT AT DIFFERENT VOLTAGES

\begin{tabular}{ccccc}
\hline \hline Voltage & \multicolumn{2}{c}{ Max. $\left(\times 10^{-6} \mathrm{~m}\right)$} & \multicolumn{2}{c}{ RMS $\left(\times 10^{-6} \mathrm{~m}\right)$} \\
$(\mathrm{V})$ & Experimental & Numerical & Experimental & Numerical \\
\hline 30 & 102.51 & 108.99 & 55.32 & 57.91 \\
70 & 273.81 & 254.32 & 139.53 & 135.12 \\
140 & 524.56 & 508.64 & 262.31 & 270.24 \\
\hline \hline
\end{tabular}

2) the feasibility and effectiveness of the proposed DMPC:

Consider a shape control target for an antenna reflector where the displacement in the Z-direction of the reflector surface should be maintained as a paraboloid with a focal length of 100 $\mathrm{m}$. The target shape is given as shown in the top right corner of Fig. 7. Herein, it is assumed that all the local MPC controllers are designed based on the prediction model at the $1^{s t}$ level grid. The weighting matrices for each subsystem are set to $\boldsymbol{Q}_{i}=$ diag $\left\{\boldsymbol{Q}_{i u}, \boldsymbol{Q}_{i v}\right\}$ and $\boldsymbol{R}_{i}=0.01 \times \boldsymbol{I}_{m i}$, where $\boldsymbol{Q}_{i u}=9 \times 10^{13} \times \boldsymbol{I}_{p i}, \boldsymbol{Q}_{i v}=$ $1 \times 10^{8} \times \boldsymbol{I}_{p i}, \boldsymbol{I}_{p i}$, and $\boldsymbol{I}_{m i}$ are $r_{i} \times r_{i}$ and $m_{i} \times m_{i}$ unit matrices, respectively. $r_{i}$ and $m_{i}$ are the number of observation nodes and PZT actuators for the $i^{\text {th }}$ subsystem, respectively. The prediction horizon $T=0.02 \mathrm{~s}$, and the number of prediction points $N=10$. The maximum number of interaction iterations is $p_{\max }=15$. The convergence error $\varepsilon=1 \times 10^{-8}$. The control inputs, i.e., the voltages of the PZT actuators, are all limited between 0-150 V. The initial conditions are $\boldsymbol{x}_{i i}=\dot{\boldsymbol{x}}_{i i}=\ddot{\boldsymbol{x}}_{i i}=\mathbf{0}$ and $\boldsymbol{u}_{i}=\mathbf{0}$.

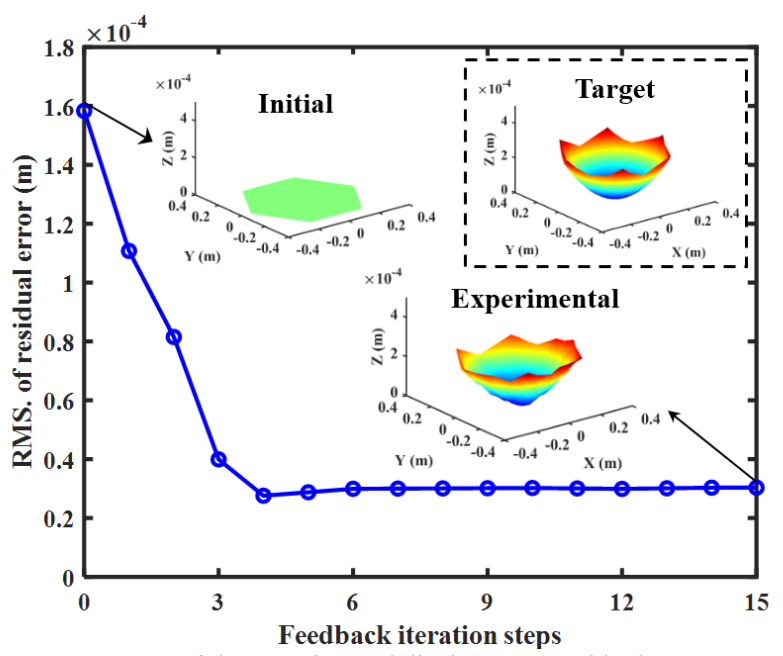

Fig. 7 RMS curve of the experimental displacement residual error 


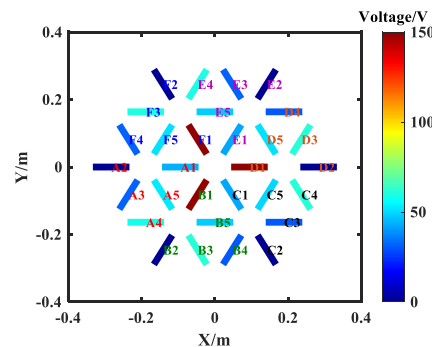

(a)

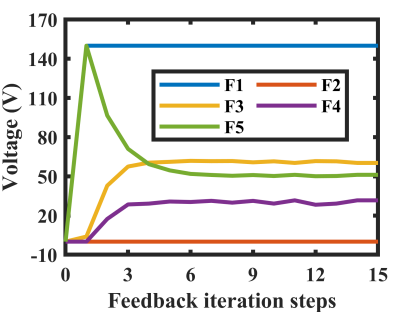

(b)
Fig. 8 The experimental voltages: (a) The voltage distribution of the system at convergence time. (b) The voltage curves for the F actuator subset

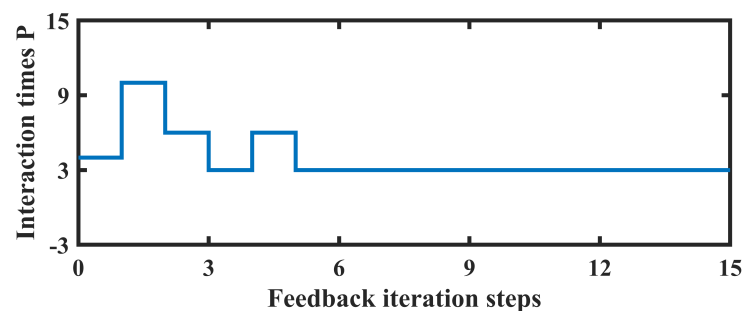

Fig. 9 The coordinated interaction times among subsystems in the experiment

First, the feasibility of the proposed DMPC method is studied in theory and hardware sampling requirements from two aspects: the existence of local optimal solutions and online computing time. For the former, based on the aforementioned reflector model and algorithm parameters, one can verify that the matrix $\boldsymbol{T}_{i}(i=1,2, \ldots, 6)$ in the LCP (49) of each subsystem is positive definite. Thus, the LCP in this work has a unique solution for all $\boldsymbol{v}_{i}$. A proof relying on an equivalent QP problem can be found in [37, p. 141]. Then, allying with the conclusion of Remark 2, a local optimal solution for each MPC controller can be obtained in theory after a finite number of interactive iterations. For the latter, as is known to all, the online computing time of a closed-loop control method must be less than the hardware sampling period. In this experiment, the average online CPU time for one step by the proposed DMPC method is $8.2 \times 10^{-3} \mathrm{~s}$, which is much smaller than the sampling period of $0.02 \mathrm{~s}$. These aforementioned results illustrate that the proposed DMPC is feasible for solving the reflector shape control problem in theory and hardware sampling requirements. Furthermore, the feasibility of the proposed DMPC can be studied more intuitively just by the control effect.

Then, to demonstrate the effectiveness of the proposed DMPC, the RMS curve of the experimental displacement residual error is shown in Fig. 7. The displacement field in the Z-direction of the reflector surface at the initial and final convergence times are also presented to show the control effect intuitively. In Fig. 7, one can see that after several closed-loop feedback iterations, the RMS of the displacement residual error is reduced from the initial value of $1.58 \times 10^{-4} \mathrm{~m}$ to a stable value of $3.03 \times 10^{-5} \mathrm{~m}$, which is reduced by approximately $80.82 \%$. The experimental control voltages of each PZT actuator subset at the convergence time are given in Fig. 8(a). As shown in Fig. 8(a), the 30 actuators are equally divided into 6 subsets; each subset is distinguished by the letters A to F. The time history curves of the voltages for the $\mathrm{F}$ actuator subset are presented in Fig. 8(b). All the control voltages in Fig. 8(b) are limited between $0-150 \mathrm{~V}$, which will satisfy the input saturation constraints. In addition, the number of coordinated interactions between the subsystems is given in Fig. 9 .

TABLE IV

RESUlts COMPARISON AMONG DiFFERENT CONTROL METHODS

\begin{tabular}{ccccc}
\hline \hline Methods & LS & PSO & CMPC & DMPC \\
\hline RMS of residual errors $\left(\times 10^{-6} \mathrm{~m}\right)$ & 69.84 & 84.77 & 26.97 & 30.32 \\
On-line CPU time $\left(\mathrm{x} 10^{-3} \mathrm{~s}\right)$ & 3.78 & 46.50 & 41.36 & 8.20 \\
\hline \hline
\end{tabular}

Before the end of this item, two other typical methods for active shape control of an antenna reflector, i.e., the least-square (LS) method [38] and particle swarm optimization (PSO) [39], are used for comparison. They can be coded directly by calling the corresponding solvers in the optimization toolbox of MATLAB software. The RMS of residual errors and the CPU time by the LS, the PSO, the CMPC, and the proposed DMPC are listed in Table IV. Based on the results in Table IV, the online CPU time for one step of the proposed DMPC is $17.63 \%$ of the PSO and $19.83 \%$ of the CMPC; its RMS of residual errors is $43.41 \%$ of the LS and $35.77 \%$ of the PSO. In addition, it can be seen that the CPU time of the proposed DMPC method is slightly larger than that of the LS, and the RMS of residual errors is also slightly larger than that of the CMPC. The former may be because the reflector used in this experiment is not large enough, and the interactive iteration between the local MPC controllers of the proposed DMPC may drag down its overall calculation time. However, if the size of the reflector or the number of inputs and outputs increase, the LS may fail to give credible solutions due to either the lack of centralized information collection ability or centralized computing ability, while the proposed DMPC can share the calculation burden just by adding more local subcontrollers. For the latter, it should be noted herein that the control accuracy for most of the distributed control strategies may not be better than that of the corresponding centralized control strategies. However, compared with CMPC, the proposed DMPC has great advantages in computational efficiency, flexibility of controller design, and fault tolerance.

Thus far, these above experimental results illustrate that the proposed DMPC algorithm is feasible and effective.

3) the flexibility of the proposed DMPC:

The flexibility of the proposed multilevel DMPC algorithm framework is mainly reflected in the following two aspects. First, taking advantage of the multilevel system decomposition mode, the MPC controller of each subsystem can be designed flexibly at different levels. Second, the interaction mode among local subsystems can also be designed flexibly.

To demonstrate the first point of flexibility, three control cases are investigated. The RMS values of the residual error for each case are listed in Table V. Based on the data of Table V, the RMS residual errors gradually decrease from case 1 to case 3. For case 3 , the RMS residual error is $27.71 \mu \mathrm{m}$, which is very close to the result of CMPC, i.e., $26.97 \mu \mathrm{m}$. This phenomenon can be explained by the fact that the higher the level at which the local controller is designed, the more mechanical information of the structure, such as the equivalent static 
stiffness for the prediction model, is condensed; thus, a better control performance can be obtained when a local MPC controller is designed based on the higher-level subsystem prediction model.

TABLE V

THE RMS OF THE RESIDUAL ERRORS FOR DIFFERENT CONTROL CASES

\begin{tabular}{cccccc}
\hline \hline $\begin{array}{c}\text { Residual } \\
\text { error }\left(\times 10^{-6} \mathrm{~m}\right)\end{array}$ & Uncontrolled & \multirow{2}{*}{ CMPC } & \multicolumn{3}{c}{ DMPC } \\
\cline { 4 - 6 } & 158.37 & 26.97 & 30.32 & 29.35 & 27.71 \\
\hline RMS & 150.92 & Case 2 & Case 3 \\
\hline \hline
\end{tabular}

Notes: Case 1: all controllers are designed at the first level; Case 2: controller No.1 is designed at the second level, and the others are designed at the first level; Case 3: all controllers are designed at the second level.

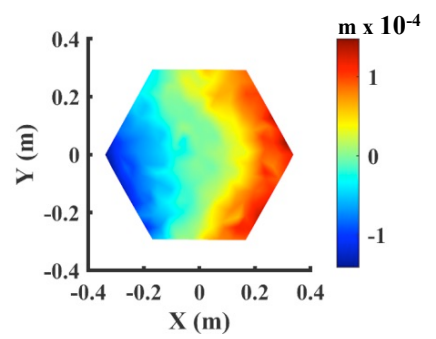

(a)

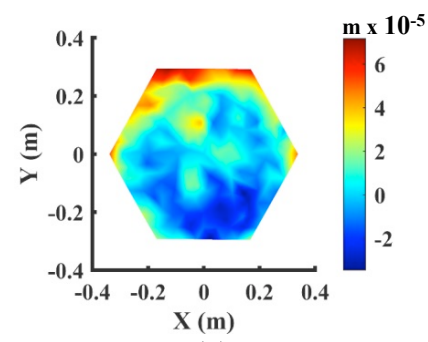

(c)

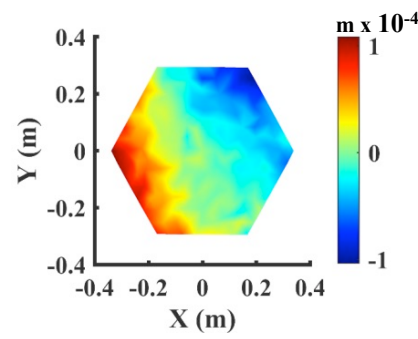

(b)

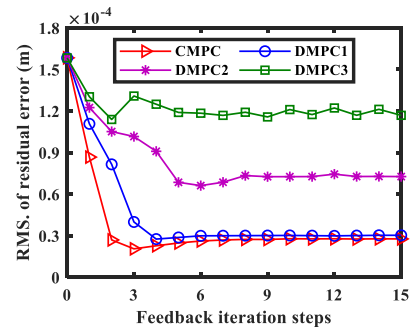

(d)
Fig. 10 The experimental control results for different interaction modes: (a)(c) The residual error distribution of DMPC1, DMPC2, and DMPC3, respectively. (d) The RMS residual errors

To demonstrate the second point of flexibility, three interaction modes among subsystems are considered, including the interaction of all subsystems, the interaction of neighboring subsystems, and ignoring the impact of all other subsystems. For the sake of concise expression, we name these modes DMPC1, DMPC2, and DMPC3. The experimental control results for the three interaction modes are presented along with those controlled by the CMPC method as shown in Fig. 10. In Fig. 10(d), it can be seen that from DMPC3 to DMPC1, the curves of the RMS residual error gradually decrease and tend to the CMPC curve. In addition, the residual error distributions of DMPC1, DMPC2, and DMPC3 are presented in Figs. 10(a-c). Thus far, the above experimental results demonstrate that the proposed DMPC method is flexible.

4) the fault tolerance of the proposed DMPC:

To illustrate the high fault tolerance of the proposed DMPC algorithm, the control performance is further studied assuming that certain local distributed controllers have failed. The RMS values of the residual displacement error with different numbers of failed local controllers are listed in Table VI.

Based on the data in Table VI, with an increasing number of failed local controllers, the maximum absolute (Abs.) and RMS values of the control residual error increase gradually and tend to those of the uncontrolled results. This illustrates that the failure of certain local controllers will reduce the systemwide control performance but will not cause the entire control system to crash suddenly. Even when half of the local controllers fail, the Max. Abs. and RMS of the residual error can still be reduced by about $12.48 \%$ and $40.29 \%$, respectively. Thus far, the above associated results show that the proposed DMPC method has high fault tolerance for the sudden failure of certain controllers.

TABLE VI

CONTROL RESUlTS FOR DiFFERENT NUMBERS OF FAILED CONTROLLERS

\begin{tabular}{cccccc}
\hline \hline \multirow{2}{*}{$\begin{array}{c}\text { Residual } \\
\text { error }\left(\times 10^{-6} \mathrm{~m}\right)\end{array}$} & \multirow{2}{*}{ Uncontrolled } & \multicolumn{3}{c}{ Number of the failed local controllers } \\
\cline { 3 - 6 } & & no failed & 1 & 1,3 & $1,2,4$ \\
\hline Max. Abs. & 289.00 & 71.87 & 178.14 & 187.96 & 252.94 \\
RMS & 158.37 & 30.32 & 72.83 & 84.94 & 94.56 \\
\hline \hline
\end{tabular}

In sum, from all the aforementioned experimental results and discussions, certain merits of the proposed DMPC algorithm framework can be concluded as follows:

Based on the substructuring technique, the overall structural system can be decomposed into multilevel subsystems with a simple and unified process; the proposed algorithm provides a flexible multilevel distributed control framework with excellent performance so that the controllers of each subsystem can be flexibly designed based on different level prediction models; the proposed algorithm has strong stability under the sudden failure of certain local controllers; and the input saturation of actuators can be satisfied directly by solving an LCP problem.

\section{Conclusions}

In this paper, we have proposed a physics-guided coordinated DMPC algorithm framework with application to structural shape control. First, the primary formulations of DMPC for a smart structural system equipped with PZT actuators are presented. Then, from a physics-guided point of view, the entire system is decomposed into multilevel subsystems based on the substructuring technique. With different prediction models at different levels, each controller can be designed flexibly. To improve the systemwide control performance, a coordinator between each subsystem is established in an iterative fashion. Finally, the control inputs for each subsystem can be obtained by transforming the original DMPC problem into a series of LCP problems; at the same time, the input saturation constraints are satisfied directly. The proposed method provides a simple and unified distributed control mode with high fault tolerance. The experimental results on the shape control of an antenna reflector structure demonstrate that the proposed DMPC algorithm is effective, flexible to design, and able to provide a control result comparable to that of a standard CMPC method. For future work, based on the physics-guided concept proposed in the current distributed control framework of linear structural systems, another new distributed control method can be further developed to extend to nonlinear structural systems.

\section{APPENDIX A}

Proof: First, at time $t_{k}$, take the value of the system cost function after $p(k)$ iterates as the Lyapunov function candidate that denoted by $V_{L}^{p(k)}(\boldsymbol{z}(k))$. Thus, when $\left(\boldsymbol{A}_{i i}, \overline{\boldsymbol{C}}_{i}\right)$ is detectable, 


$$
\begin{aligned}
V_{L}^{p(k)}(\boldsymbol{z}(k)) & =\Phi\left(\boldsymbol{u}_{1}^{p(k)}, \boldsymbol{u}_{2}^{p(k)}, \cdots, \boldsymbol{u}_{S}^{p(k)} ; \boldsymbol{z}(k)\right) \\
& =\sum_{i=1}^{S} \omega_{i} \sum_{q=0}^{N} J_{i}\left(\boldsymbol{z}_{i}^{p(k)}(k+q \mid k), \boldsymbol{u}_{i}^{p(k)}(k+q \mid k)\right)
\end{aligned}
$$

where

$$
J_{i}\left(\boldsymbol{z}_{i}(t \mid k), \boldsymbol{u}_{i}(t \mid k)\right)=\frac{1}{2}\left(\left\|\boldsymbol{z}_{i}(t \mid k)-\boldsymbol{z}_{i d}\right\|_{\boldsymbol{Q}_{i}}^{2}+\left(\left\|u_{i}(t \mid k)\right\|_{\boldsymbol{R}_{i}}^{2}\right)\right.
$$

In addition, let $V_{L}^{0}(z(k))$ represent the cost function value with the initial input trajectory $\boldsymbol{u}_{i}^{0}(k)$, defined as $\boldsymbol{u}_{i}^{0}(k)=\boldsymbol{u}_{i}^{p(k-1)}(k-1)$. Then, from the definition of $\boldsymbol{u}_{i}^{p}$ in Algorithm 1, we have

$$
\begin{aligned}
& \Phi\left(\boldsymbol{u}_{1}^{p}, \boldsymbol{u}_{2}^{p}, \cdots, \boldsymbol{u}_{S}^{p} ; z(k)\right) \\
& =\Phi\left(\omega_{1} \boldsymbol{u}_{1}^{p(*)}+\left(1-\omega_{1}\right) \boldsymbol{u}_{1}^{p-1}, \cdots, \omega_{S} \boldsymbol{u}_{S}^{p(*)}+\left(1-\omega_{S}\right) \boldsymbol{u}_{S}^{p-1} ; z(k)\right) \\
& \text { By } \sum_{i=1}^{S} \omega_{i}=1, \\
& =\Phi\left(\omega_{1} \boldsymbol{u}_{1}^{p\left(^{*}\right)}+\omega_{2} \boldsymbol{u}_{1}^{p-1}+\cdots+\omega_{S} \boldsymbol{u}_{1}^{p-1}, \omega_{1} \boldsymbol{u}_{2}^{p-1}+\omega_{2} \boldsymbol{u}_{2}^{p(*)}+\cdots+\omega_{S} \boldsymbol{u}_{2}^{p-1},\right. \\
& \left.\quad \cdots, \omega_{1} \boldsymbol{u}_{S}^{p-1}+\omega_{2} \boldsymbol{u}_{S}^{p-1}+\cdots+\omega_{S} \boldsymbol{u}_{S}^{p(*)} ; z(k)\right)
\end{aligned}
$$

By convexity of $\Phi(\cdot)$

$$
\begin{aligned}
& \leq \sum_{i=1}^{S} \omega_{i} \Phi\left(\boldsymbol{u}_{1}^{p-1}, \cdots, \boldsymbol{u}_{i-1}^{p-1}, \boldsymbol{u}_{i}^{p(*)}, \boldsymbol{u}_{i+1}^{p-1}, \cdots, \boldsymbol{u}_{S}^{p-1} ; \boldsymbol{z}(k)\right) \\
& \leq \sum_{i=1}^{S} \omega_{i} \Phi\left(\boldsymbol{u}_{1}^{p-1}, \cdots, \boldsymbol{u}_{i-1}^{p-1}, \boldsymbol{u}_{i}^{p-1}, \boldsymbol{u}_{i+1}^{p-1}, \cdots, \boldsymbol{u}_{S}^{p-1} ; \boldsymbol{z}(k)\right) \\
& =\Phi\left(\boldsymbol{u}_{1}^{p-1}, \boldsymbol{u}_{2}^{p-1}, \cdots, \boldsymbol{u}_{S}^{p-1} ; \boldsymbol{z}(k)\right)
\end{aligned}
$$

in which equality is obtained if $\boldsymbol{u}_{i}^{p}=\boldsymbol{u}_{i}^{p-1}$. Equation (A3) illustrates that the sequence of cost functions $\left\{\Phi\left(\boldsymbol{u}_{1}^{p}, \boldsymbol{u}_{2}^{p}, \ldots\right.\right.$, $\left.\left.\boldsymbol{u}_{S}^{p} ; \boldsymbol{z}(k)\right)\right\}$ generated by Algorithm 1 is nonincreasing with the number of iterations $p$. Therefore, we have

$$
\begin{aligned}
V_{L}^{p(k+1)} & (\boldsymbol{z}(k+1))-V_{L}^{p(k)}(\boldsymbol{z}(k)) \\
& \leq V_{L}^{0}(\boldsymbol{z}(k+1))-V_{L}^{p(k)}(\boldsymbol{z}(k)) \\
& =-\sum_{i=1}^{S} \omega_{i} J_{i}\left(\boldsymbol{z}_{i}(k), \boldsymbol{u}_{i}^{p(k)}\left(k ; \boldsymbol{z}_{i}(k)\right)\right) \leq 0
\end{aligned}
$$

According to the Lyapunov stability theory [36], the origin of the system is stable. The proof is completed.

\section{APPENDIX B}

TABLE VII

PARAMETERS IN THE THEORETICAL DERIVATION FORMULA

\begin{tabular}{ll}
\hline \hline Parameters & Definition \\
\hline$\gamma$ and $\beta$ & coefficients of Rayleigh damping \\
$S$ & number of subsystems for the entire system \\
$S_{l}$ & number of subsystems in the $l^{\text {th }}$ level \\
$h$ & number of levels \\
$\boldsymbol{Q}_{i}$ and $\boldsymbol{R}_{i}$ & weighting matrices for the $i^{\text {th }}$ subsystem \\
$\overline{\boldsymbol{C}}_{i}$ & output coefficient matrix for the $i^{\text {th }}$ subsystem \\
$\alpha$ and $\delta$ & Newmark- $\beta$ algorithm parameters \\
$T$ & prediction time horizon \\
$N$ & number of prediction steps \\
$\eta$ & prediction step length \\
$\omega i$ & combination coefficients in the cost function \\
$\overline{\boldsymbol{\alpha}}_{i}$ and $\underline{\boldsymbol{\alpha}}_{i}$ & relaxation factors of inequality constraints \\
$\overline{\boldsymbol{\lambda}}_{i}$ and $\underline{\boldsymbol{\lambda}}_{i}$ & nonnegative Lagrange multipliers \\
$p$ & iteration times between local MPC controllers \\
$p_{\max }$ & maximum iteration times \\
$\varepsilon_{i}$ & convergence accuracy of iteration \\
\hline \hline
\end{tabular}

\section{REFERENCES}

[1] H. Irschik, "A review on static and dynamic shape control of structures by piezoelectric actuation," Eng. Struct., vol. 24, no. 1, pp. 5-11, Jan, 2002.

[2] R. T. Haftka, and H. M. Adelman, "An Analytical Investigation of Shape Control of Large Space Structures by Applied Temperatures," AIAA J., vol. 23, no. 3, pp. 450-457, 1985.

[3] X. Song, S. Tan, E. Wang et al., "Active shape control of an antenna reflector using piezoelectric actuators," J. Intell. Mater. Syst. Struct., vol. 30, no. 18-19, pp. 2733-2747, 2019.

[4] Y.Xie, H. Shi, A. Alleyne et al., "Feedback shape control for deployable mesh reflectors using gain scheduling method," Acta Astronaut., vol. 121, pp. 241-255, 2016

[5] T. Li, M. Fu, L. Xie et al., "Distributed consensus with limited communication data rate," IEEE Trans. Autom. Control, vol. 56, no. 2, pp. 279-292, 2011.

[6] T. Li, Z. Wang, J. Li et al., "Distributed vibration control of tensegrity structure," J. Vib. Control, vol. 19, no. 5, pp. 720-728, 2012.

[7] X. Chen, M. Shi, J. Zhou et al., "Consensus-based distributed control for photovoltaic-battery units in a DC microgrid," IEEE Trans. on Ind. Electron., vol. 66, no. 10, pp. 7778-7787, 2019.

[8] Y. Zheng, S. Li, and H. Qiu, "Networked coordination-based distributed model predictive control for large-scale system," IEEE Trans. Control Syst. Technol., vol. 21, no. 3, pp. 991-998, 2013.

[9] Shih-Ho Wang, E.J. Davison, "On the stabilization of decentralized control systems," IEEE Trans. Autom. Control, vol. 18, no. 5, pp. 473478, 1973

[10] B. Bamieh, F. Paganini, and M.A. Dahleh, "Distributed control of spatially invariant systems," IEEE Trans. Autom. Control, vol. 47, no. 7, pp. 1091-1107, 2002.

[11] H. Zhang, T. Feng, G. H. Yang, et al., "Distributed cooperative optimal control for multiagent systems on directed graphs: An inverse optimal approach," IEEE Trans. Cybern., vol. 45, no. 7, pp. 1315-26, Jul, 2015.

[12] Q. Shen, P. Shi, and Y. Shi, "Distributed adaptive fuzzy control for nonlinear multiagent systems via sliding mode observers," IEEE Trans. Cybern., vol. 46, no. 12, pp. 3086-3097, Dec, 2016.

[13] F. Borrelli, and T. Keviczky, "Distributed LQR design for identical dynamically decoupled systems," IEEE Trans. Autom. Control, vol. 53, no. 8, pp. 1901-1912, 2008.

[14] M. Liu, Y. Shi, and X. Liu, "Distributed MPC of aggregated heterogeneous thermostatically controlled loads in smart grid," IEEE Trans. on Ind. Electron., vol. 63, no. 2, pp. 1120-1129, 2016.

[15] V. Narayanan, and S. Jagannathan, "Event-triggered distributed control of nonlinear interconnected systems using online reinforcement learning with exploration," IEEE Trans. Cybern., vol. 48, no. 9, pp. 2510-2519, Sep, 2018.

[16] S. J. Qin, and T. A. Badgwell, "A survey of industrial model predictive control technology," Control Eng. Pract., vol. 11, no. 7, pp. 733-764, 2003.

[17] M. Morari, and J. H. Lee, "Model predictive control: past, present and future," Comput. Chem. Eng., vol. 23, no. 4-5, pp. 667-682, May 1, 1999.

[18] L. Dong, J. Yan, X. Yuan et al., "Functional nonlinear model predictive control based on adaptive dynamic programming," IEEE Trans. Cybern., vol. 49, no. 12, pp. 4206-4218, Dec, 2019

[19] R. Scattolini, "Architectures for distributed and hierarchical model predictive control - A review," J. Process Control, vol. 19, no. 5, pp. 723-731, 2009.

[20] E. Camponogara, D. Jia, B. H. Krogh et al., "Distributed model predictive control," IEEE Control Syst. Mag., vol. 22, no. 1, pp. 44-52, Feb, 2002.

[21] C. Conte, C. N. Jones, M. Morari, et al., "Distributed synthesis and stability of cooperative distributed model predictive control for linear systems," Automatica, vol. 69, pp. 117-125, 2016.

[22] M. Heidarinejad, J. Liu, D. Muñoz de la Peña et al., "Multirate Lyapunov-based distributed model predictive control of nonlinear uncertain systems," J. Process Control, vol. 21, no. 9, pp. 1231-1242, 2011.

[23] B. Picasso, D. De Vito, R. Scattolini et al., "An MPC approach to the design of two-layer hierarchical control systems," Automatica, vol. 46, no. 5, pp. 823-831, 2010.

[24] J. S. Przemieniecki, "Matrix structural analysis of substructures," AIAA J., vol. 1, no. 1, pp. 138-147, 1963. 
[25] D. D. Klerk, D. J. Rixen, and S. N. Voormeeren, "General framework for dynamic substructuring: history, review and classification of techniques," AIAA J., vol. 46, no. 5, pp. 1169-1181, 2008.

[26] S. Kocak, and H. U. Akay, "Parallel Schur complement method for largescale systems on distributed memory computers," Appl. Math. Model., vol. 25, no. 10, pp. 873-886, Oct, 2001.

[27] H. Peng, F. Li, and Z. Kan, "A novel distributed model predictive control method based on a substructuring technique for smart tensegrity structure vibrations," J. Sound Vib., vol. 471, Apr 14, 2020.

[28] P. D. Christofides, R. Scattolini, D. Muñoz de la Peña et al., "Distributed model predictive control: A tutorial review and future research directions," Comput. Chem. Eng., vol. 51, pp. 21-41, 2013.

[29] R. W. Clough and J. Penzien, Dynamics of Structures, New York, USA: McGraw-Hill Companies, 1975.

[30] G. Takács, B. Rohal'-Ilkiv, Model Predictive Vibration Control: Efficient Constrained MPC Vibration Control for Lightly Damped Mechanical Structures, New York, USA: Springer-Verlag, 2012.

[31] H. Peng, F. Li, S. Zhang et al., "A novel fast model predictive control with actuator saturation for large-scale structures," Comput. Struct., vol. 187, pp. 35-49, Jul 15, 2017.

[32] A. N. Venkat, I. A. Hiskens, J. B. Rawlings et al., "Distributed MPC strategies with application to power system automatic generation control," IEEE Trans. Control Syst. Technol., vol. 16, no. 6, pp. 11921206, 2008.

[33] A. N. Venkat, J. B. Rawlings, and S. J. Wright, "Stability and optimality of distributed model predictive control," in Proc. 44th IEEE Conf. Decis. Control, Seville, Spain, 2005, pp. 6680-6685.

[34] W. X. Zhong, and R. L. Zhang, "Parametric variational-principles and their quadratic-programming solutions in plasticity," Comput. Struct., vol. 30, no. 4, pp. 887-896, 1988.

[35] S. C. Billups, K. G. Murty, "Complementarity problems," J. Comput. Appl. Math., vol. 124, pp. 303-318, 2000.

[36] Andrea Bacciotti and Lionel Rosier, Liapunov Functions and Stability in Control Theory, 2nd ed., New York, USA: Springer, 2005.

[37] R. W. Cottle, J. Pang, and R. E. Stone, The Linear Complementarity Problem, Boston, USA: Academic Press, 1992.

[38] J. Hill, K. W. Wang, and H. Fang, "Advances of Surface Control Methodologies for Flexible Space Reflectors," J. Spacecr. Rockets, vol. 50, no. 4, pp. 816-828, 2013.

[39] S. Shao, S. Song, M. Xu et al., "Mechanically reconfigurable reflector for future smart space antenna application," Smart Mater. Struct., vol. 27 , no. 9, 2018.

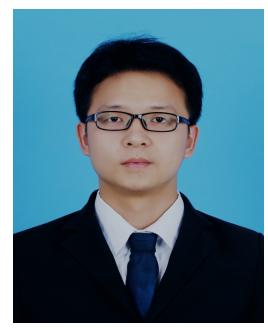

Fei Li (S'20) was born in Jiangxi, China. He received the M.S. degree in computational mechanics from Dalian University of Technology, Dalian, China in 2017. He is currently pursuing the Ph.D. degree in the Department of Engineering Mechanics, Dalian University of Technology, Dalian, China.

His current research interests include robot multibody dynamics and trajectory tracking control, distributed model predictive control, and soft tensegrity robot motion analysis and control.

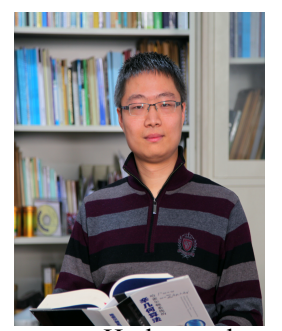

Haijun Peng (M'15) was born in Handan, China. He received the Ph.D. degree in dynamics and control from Dalian University of Technology, China in 2012.

Dr. Peng is currently a Professor with the Department of Engineering Mechanics and fixed personnel in State Key Laboratory of Structural Analysis for Industrial Equipment, Dalian University of Technology. His research interests cover the optimal control, multibody dynamics, and robot system. He has authored or co-authored over 50 publications in journals and received one best paper award.

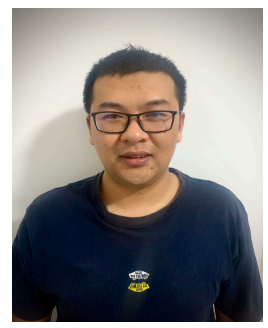

Xiangshuai Song was born in Liaoning, China. He received the M.S. degrees in the school of aeronautics and astronautics from the Dalian University of Technology, Dalian, China in 2016. He is currently pursuing the Ph.D. degree in the School of Aeronautics and Astronautics from the Dalian University of Technology, Dalian, China.

His current research interests include shape control for antenna reflectors and vibration control of smart large-scale structures.

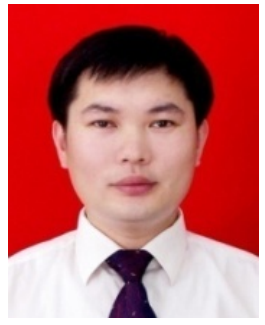

Jinguo Liu (M'07-SM'18) received his Ph.D. degrees in Mechatronics from Shenyang Institute of Automation (SIA), Chinese Academy of Sciences (CAS) in 2007. Since January 2011, he has been a Full Professor with SIA, CAS. In addition, he has held the Assistant Director position of State Key Laboratory of Robotics since 2008 and the Associate Director position of Center for Space Automation Technologies and Systems since 2015. His research interests include bio-inspired robotics and space robotics. He has authored/co-authored three books, over one hundred papers, and fifty patents in these areas. He was awarded the T. J. TARN Best Paper Award in Robotics from 2005 IEEE International Conference on Robotics and Biomimetics, the Best Paper Award of the Chinese Mechanical Engineering Society in 2007, the Best Paper Nomination Award from 2008 International Symposium on Intelligent Unmanned Systems, the Best Paper Award from 2016 China Manned Space Academic Conference, and the Outstanding Paper Award from 2017 International Conference on Intelligent Robotics and Applications, and the Best Paper Award from 2018 International Conference on Electrical Machines and systems. He is a senior member of the IEEE, IEEE Technical Committee on Safety, Security, and Rescue Robotics, IEEE Technical Committee on Marine Robotics, and the Senior Member of Chinese Mechanical Engineering Society.

Dr. Liu services as the Associate Editor or Technical Editor of several journals such as IEEE/ASME Transactions on Mechatronics, Mechanical Sciences, Science China Technological Sciences, Chinese Journal of Mechanical Engineering, and Chinese Journal of Aeronautics.

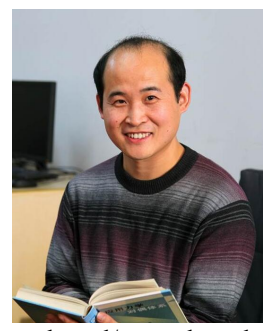

Shujun Tan was born in Shandong, China. He received the Ph.D. degree in dynamics and control from Dalian University of Technology, China in 2009.

$\mathrm{He}$ is currently an Associate Professor in the School of Aeronautics and Astronautics, Dalian University of Technology. His research interests cover the aircraft dynamics and control, space intelligent structure design, analysis and control, system identification, and optimal control. He has authored/co-authored over 50 publications in journals and 2 books.

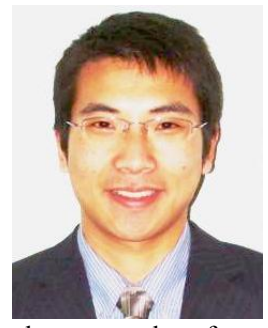

Zhaojie Ju (M'08-SM'16) received the B.S. degree in automatic control and the M.S. degree in intelligent robotics from the Huazhong University of Science and Technology, China, and the Ph.D. degree in intelligent robotics from the University of Portsmouth, U.K. He held research appointments at University College London, London, U.K., before he started his independent academic position at the University of Portsmouth, in 2012. He has authored or coauthored over 200 publications in journals, book chapters, and conference proceedings and received five Best Paper Awards and one Best AE Award in ICRA2018. His research interests include machine intelligence, pattern recognition and their applications on human motion analysis, multi-fingered robotic hand control, human-robot interaction and collaboration, and robot skill learning.

Dr. Ju is an Associate Editor of several journals, such as IEEE TRANSACTIONS ON CYBERNETICS, IEEE TRANSACTIONS ON COGNITIVE AND DEVELOPMENTAL SYSTEMS and Neurocomputing. 